\title{
A Tutorial on Parallel and Concurrent Programming in Haskell
}

\author{
Simon Peyton Jones and Satnam Singh \\ Microsoft Research Cambridge \\ simonpj@microsoft.com satnams@microsoft.com
}

\begin{abstract}
This practical tutorial introduces the features available in Haskell for writing parallel and concurrent programs. We first describe how to write semi-explicit parallel programs by using annotations to express opportunities for parallelism and to help control the granularity of parallelism for effective execution on modern operating systems and processors. We then describe the mechanisms provided by Haskell for writing explicitly parallel programs with a focus on the use of software transactional memory to help share information between threads. Finally, we show how nested data parallelism can be used to write deterministically parallel programs which allows programmers to use rich data types in data parallel programs which are automatically transformed into flat data parallel versions for efficient execution on multi-core processors.
\end{abstract}

\section{Introduction}

The introduction of multi-core processors has renewed interest in parallel functional programming and there are now several interesting projects that explore the advantages of a functional language for writing parallel code or implicitly paralellizing code written in a pure functional language. These lecture notes present a variety of techniques for writing concurrent parallel programs which include existing techniques based on semi-implicit parallelism and explicit thread-based parallelism as well as more recent developments in the areas of software transactional memory and nested data parallelism.

We also use the terms parallel and concurrent with quite specific meanings. A parallel program is one which is written for performance reasons to exploit the potential of a real parallel computing resource like a multi-core processor. For a parallel program we have the expectation of some genuinely simultaneous execution. Concurrency is a software structuring technique that allows us to model computations as hypothetical independent activities (e.g. with their own program counters) that can communicate and synchronize.

In these lecture notes we assume that the reader is familiar with the pure lazy functional programming language Haskell. 


\section{Applications of concurrency and parallelism}

Writing concurrent and parallel programs is more challenging than the already difficult problem of writing sequential programs. However, there are some compelling reasons for writing concurrent and parallel programs:

Performance. We need to write parallel programs to achieve improving performance from each new generation of multi-core processors.

Hiding latency. Even on single-core processors we can exploit concurrent programs to hide the latency of slow I/O operations to disks and network devices.

Software structuring. Certain kinds of problems can be conveniently represented as multiple communicating threads which help to structure code in a more modular manner e.g. by modeling user interface components as separate threads.

Real world concurrency. In distributed and real-time systems we have to model and react to events in the real world e.g. handling multiple server requests in parallel.

All new mainstream microprocessors have two or more cores and relatively soon we can expect to see tens or hundreds of cores. We can not expect the performance of each individual core to improve much further. The only way to achieve increasing performance from each new generation of chips is by dividing the work of a program across multiple processing cores. One way to divide an application over multiple processing cores is to somehow automatically parallelize the sequential code and this is an active area of research. Another approach is for the user to write a semi-explicit or explicitly parallel program which is then scheduled onto multiple cores by the operating systems and this is the approach we describe in these lectures.

\section{Compiling Parallel Haskell Programs}

To reproduce the results in this paper you will need to use a version of the GHC Haskell compiler later than 6.10 .1 (which at the time of printing requires building the GHC compiler from the HEAD branch of the source code repository). To compile a parallel Haskell program you need to specify the -threaded extra flag. For example, to compile the parallel program contained in the file Wombat.hs issue the command:

ghc --make -threaded Wombat.hs

To execute the program you need to specify how many real threads are available to execute the logical threads in a Haskell program. This is done by specifying an argument to Haskell's run-time system at invocation time. For example, to use three real threads to execute the Wombat program issue the command:

Wombat +RTS -N3

In these lecture notes we use the term thread to describe a Haskell thread rather than a native operating system thread. 


\section{Semi-Explicit Parallelism}

A pure Haskell program may appear to have abundant opportunities for automatic parallelization. Given the lack of side effects it may seem that we can productively evaluate every sub-expression of a Haskell program in parallel. In practice this does not work out well because it creates far too many small items of work which can not be efficiently scheduled and parallelism is limited by fundamental data dependencies in the source program.

Haskell provides a mechanism to allow the user to control the granularity of parallelism by indicating what computations may be usefully carried out in parallel. This is done by using functions from the Control.Parallel module. The interface for Control.Parallel is shown below:

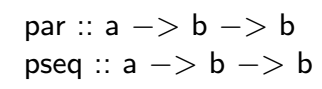

The function par indicates to the Haskell run-time system that it may be beneficial to evaluate the first argument in parallel with the second argument. The par function returns as its result the value of the second argument. One can always eliminate par from a program by using the following identity without altering the semantics of the program:

1 par $a b=b$

The Haskell run-time system does not necessarily create a thread to compute the value of the expression a. Instead, the run-time system creates a spark which has the potential to be executed on a different thread from the parent thread. A sparked computation expresses the possibility of performing some speculative evaluation. Since a thread is not necessarily created to compute the value of a this approach has some similarities with the notion of a lazy future [1].

Sometimes it is convenient to write a function with two arguments as an infix function and this is done in Haskell by writing quotes around the function:

1 a 'par' b

An example of this expression executing in parallel is shown in Figure1.

We call such programs semi-explicitly parallel because the programmer has provided a hint about the appropriate level of granularity for parallel operations and the system implicitly creates threads to implement the concurrency. The user does not need to explicitly create any threads or write any code for inter-thread communication or synchronization.

To illustrate the use of par we present a program that performs two compute intensive functions in parallel. The first compute intensive function we use is the notorious Fibonacci function:

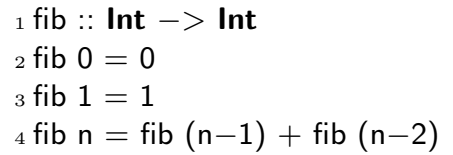

The second compute intensive function we use is the sumEuler function [2]: 


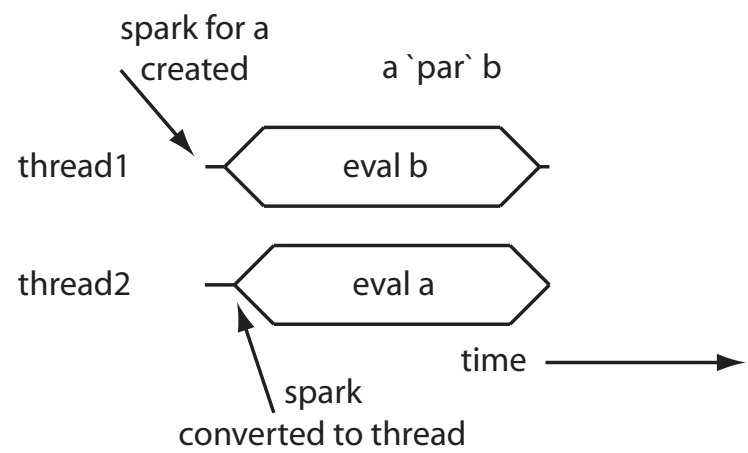

Fig. 1. Semi-explicit execution of a in parallel with the main thread b

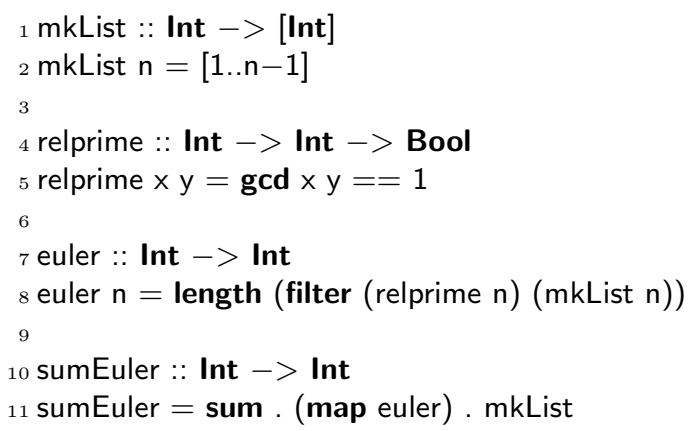

The function that we wish to parallelize adds the results of calling fib and sumEuler:

1 sumFibEuler :: Int $->$ Int $->$ Int

${ }_{2}$ sumFibEuler $a b=$ fib $a+$ sumEuler $b$

As a first attempt we can try to use par the speculatively spark off the computation of fib while the parent thread works on sumEuler:

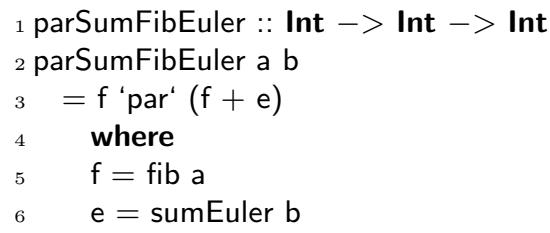

To help measure how long a particular computation is taking we use the Sytem. Time module and define a function that returns the difference between two time samples as a number of seconds:

1 secDiff :: ClockTime $->$ ClockTime $->$ Float

2 secDiff (TOD secs1 psecs1) (TOD secs2 psecs2) 
$3=$ fromlnteger $($ psecs $2-$ psecs 1$) / 1 \mathrm{e} 12+$ fromlnteger $(\operatorname{secs} 2-\operatorname{secs} 1)$

The main program calls the sumFibEuler function with suitably large arguments and reports the value

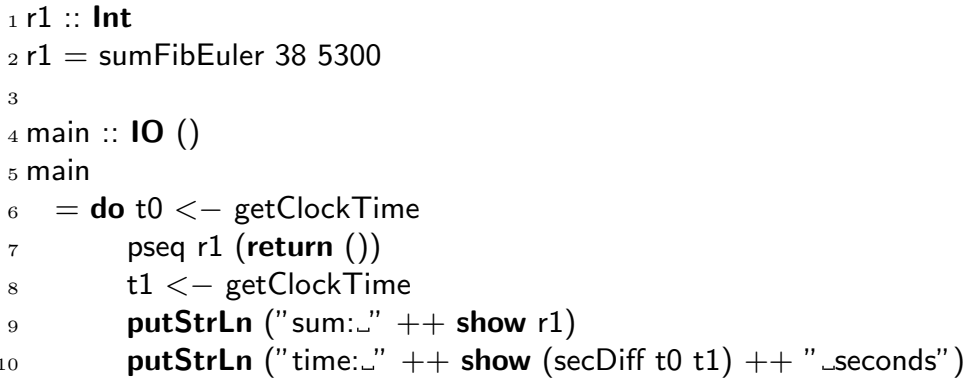

The calculations fib 38 and sumEuler 5300 have been chosen to have roughly the same execution time.

If we were to execute this code using just one thread we would observe the sequence of evaluations shown in Figure 2. Although a spark is created for the evaluation of $f$ there is no other thread available to instantiate this spark so the program first computes $f$ (assuming the + evaluates its left argument first) and then computes e and finally the addition is performed. Making an assumption about the evaluation order of the arguments of + is unsafe and another valid execution trace for this program would involve first evaluating e and then evaluating $f$.

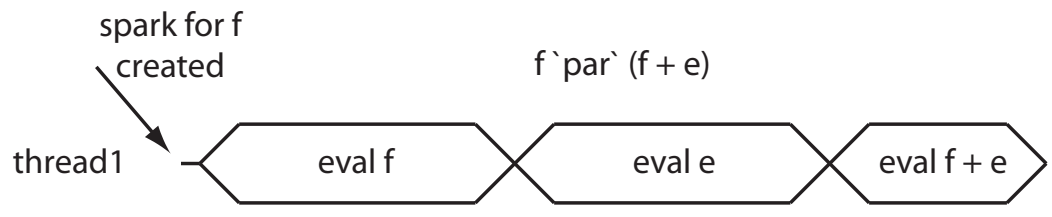

Fig. 2. Executing $f$ 'par' $(e+f)$ on a single thread

The compiled program can now be run on a multi-core computer and we can see how it performs when it uses one and then two real operating system threads:

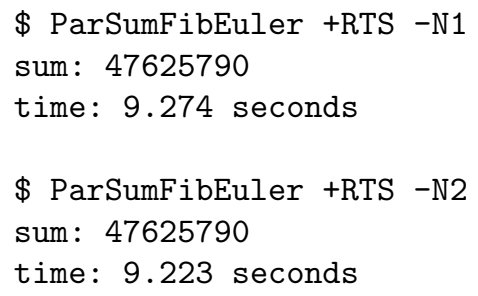


The output above shows that the version run with two cores did not perform any better than the sequential version. Why is this? The problem lies in line 3 of the parSumFibEuler function. Although the work of computing fib 38 is sparked off for speculative evaluation the parent thread also starts off by trying to compute fib 38 because this particular implementation of the program used a version of + that evaluates its left and side before it evaluates its right hand side. This causes the main thread to demand the evaluation of fib 38 so the spark never gets instantiated onto a thread. After the main thread evaluates fib 38 it goes onto evaluate sumEuler 5300 which results in a performance which is equivalent to the sequential program. A sample execution trace for this version of the program is shown in Figure 3. We can obtain further information about

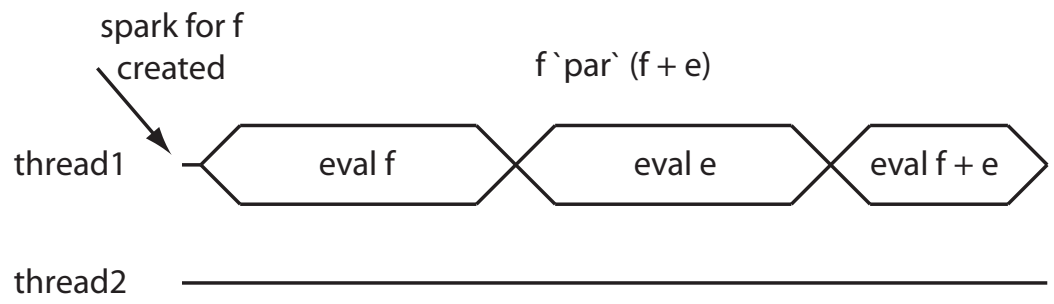

Fig. 3. A spark that does not get instantiated onto a thread

what happened by asking the run-time system to produce a log which contains information about how many sparks were created and then actually evaluated as well as information about how much work was performed by each thread. The -s flag by itself will write out such information to standard output or it can be followed by the name of a $\log$ file.

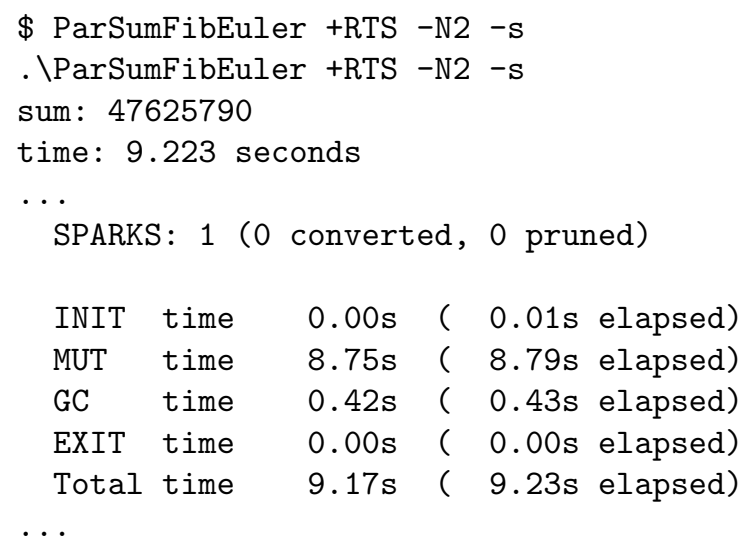

This report shows that although a spark was created it was never actually taken up for execution on a thread. This behaviour is also reported when we view the execution trace using the ThreadScope thread profiler as shown in Figure 4 
which shows one thread busy all the time but the second thread performs no work at all. Purple (or black) indicates that a thread is running and orange (or gray) indicates garbage collection.

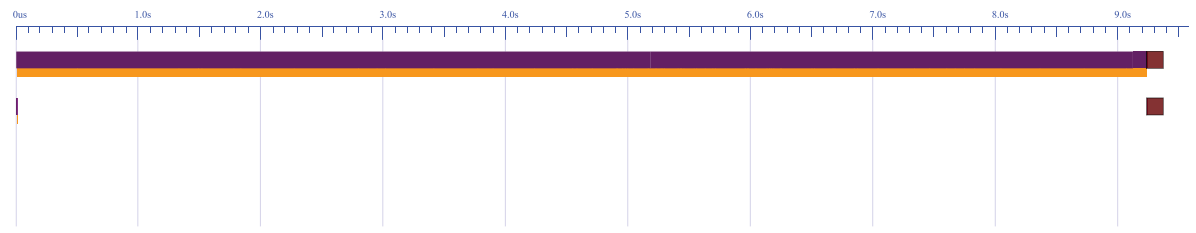

Fig. 4. A ThreadScope trace showing lack of parallelism

A tempting fix is to reverse the order of the arguments to + :

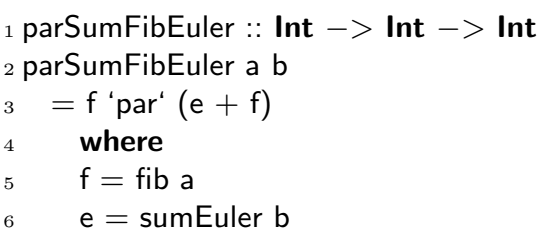

Here we are sparking off the computation of fib for speculative evaluation with respect to the parent thread. The parent thread starts off by computing sumEuler and hopefully the run-time will convert the spark for computing fib and execute it on a thread located on a different core in parallel with the parent thread. This does give a respectable speedup:

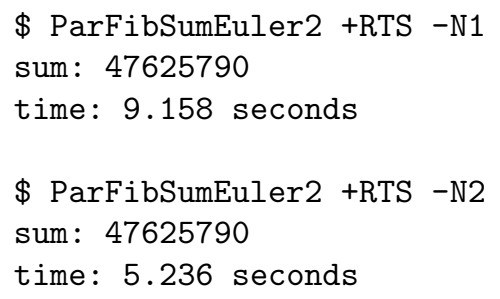

A sample execution trace for this version of the program is shown in Figure 5

We can confirm that a spark was created and productively executed by looking at the log output using the -s flag:

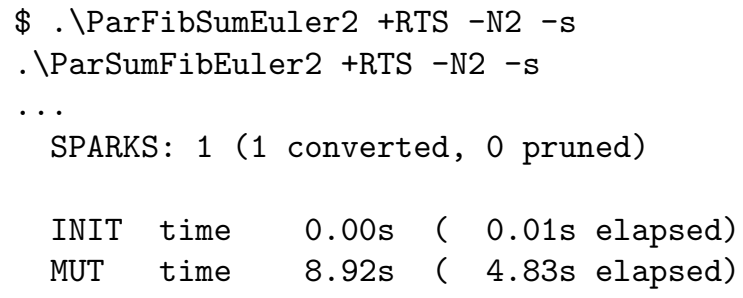




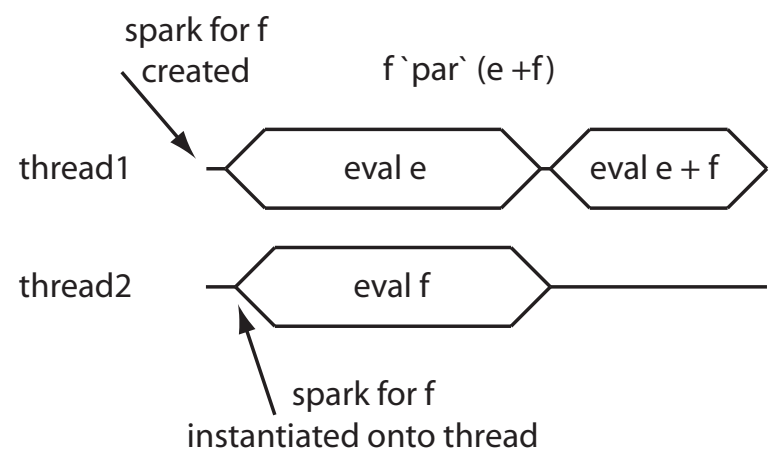

Fig. 5. A lucky parallelization (bad dependency on the evaluation order of + )

$\begin{array}{lllll}\text { GC } & \text { time } & 0.39 \mathrm{~s} & (0.41 \mathrm{~s} \text { elapsed }) \\ \text { EXIT time } & 0.00 \mathrm{~s} & (0.00 \mathrm{~s} \text { elapsed }) \\ \text { Total time } & 9.31 \mathrm{~s} & (5.25 \mathrm{~s} \text { elapsed })\end{array}$

...

Here we see that one spark was created and converted into work for a real thread. A total of 9.31 seconds worth of work was done in 5.25 seconds of wall clock time indicating a reasonable degree of parallel execution. A ThreadScope trace of this execution is shown in Figure 6 which clearly indicates parallel activity on two threads.

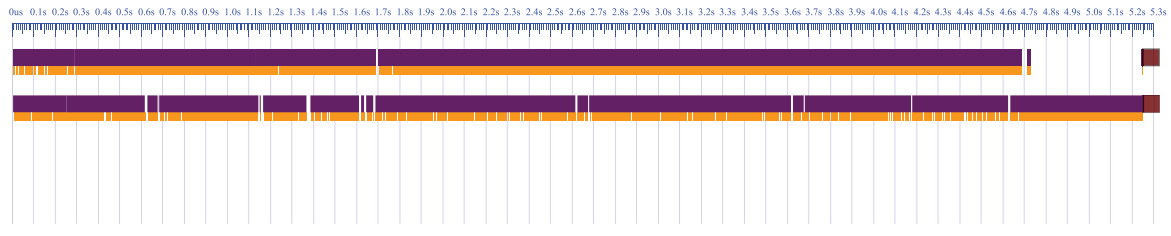

Fig. 6. A ThreadScope trace showing a lucky parallelization

However, it is a Very Bad Idea to rely on the evaluation order of + for the performance (or correctness) of a program. The Haskell language does not define the evaluation order of the left and right hand arguments of + and the compiler is free to transform $a+b$ to $b+a$. What we really need to be able to specify what work the main thread should do first. We can use the pseq function from the Control.Monad module for this purpose. The expression a 'pseq' b evaluates $a$ and then returns $b$. We can use this function to specify what work the main thread should do first (as the first argument of pseq) and we can then return the 
result of the overall computation in the second argument without worrying about things like the evaluation order of + . This is how we can re-write ParFibSumEuler with pseq:

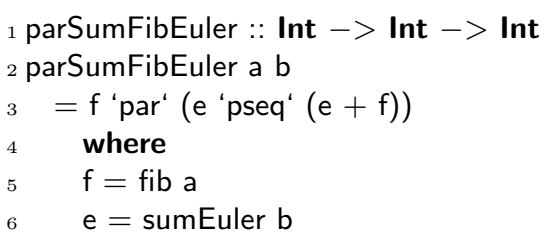

This program still gives a roughly $2 \mathrm{X}$ speedup as does the following version which has the arguments to + reversed but the use of pseq still ensures that the main thread works on sumEuler before it computes fib (which will hopefully have been computed by a speculatively created thread):

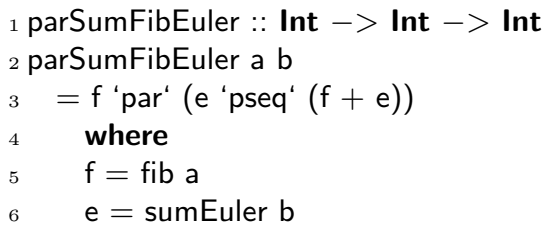

An execution trace for this program is shown in Figure 7.

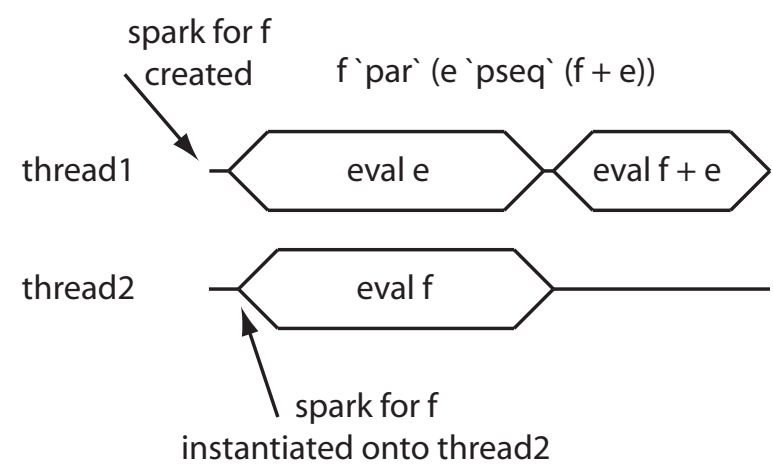

Fig. 7. A correct parallelization which is not dependent on the evaluation order of +

\subsection{Weak Head Normal Form (WHNF)}

The program below is a variant of the fib-Euler program in which each parallel workload involves mapping an operation over a list. 


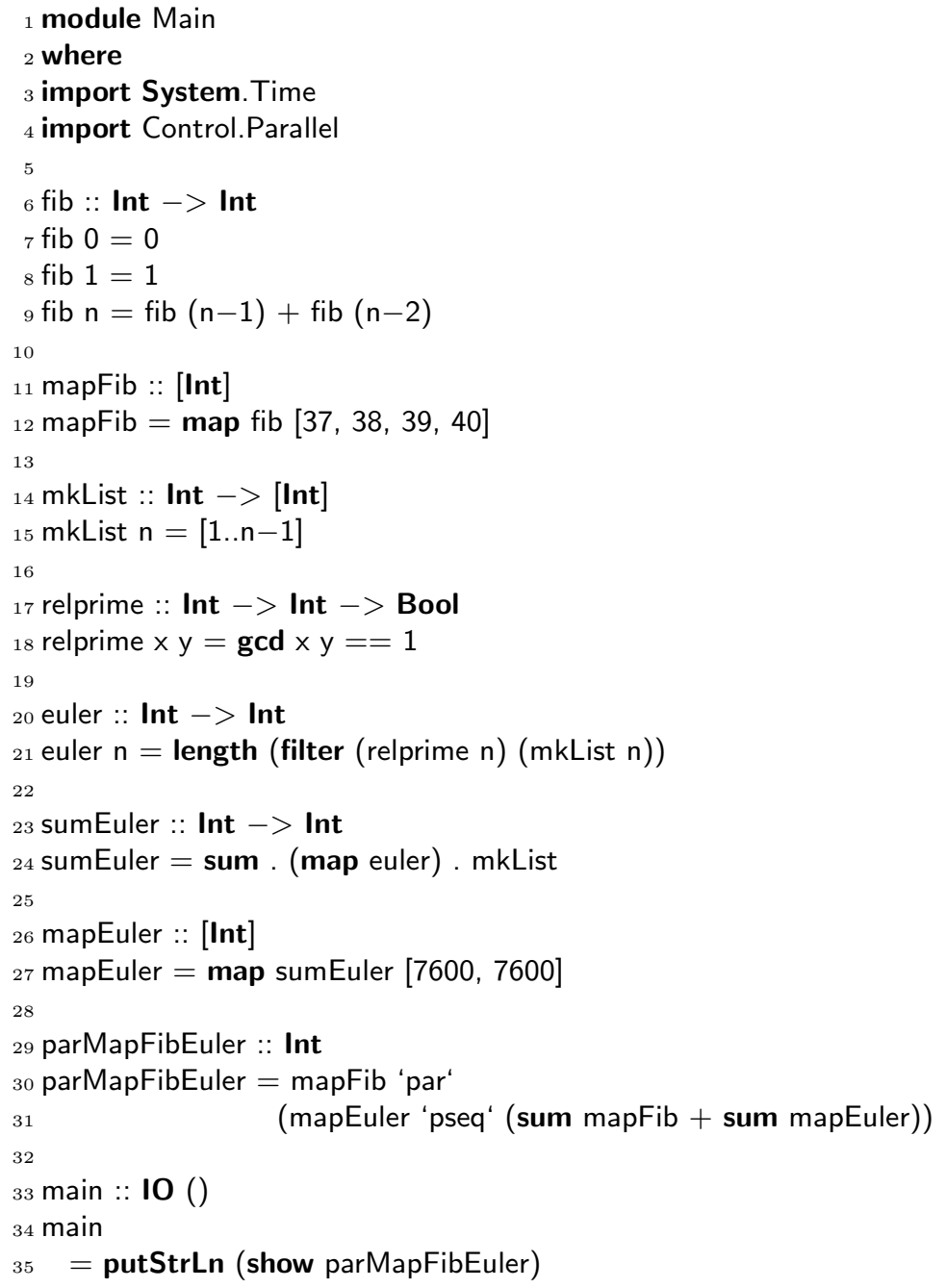

The intention here is to compute two independent functions in parallel:

- mapping the fib function over a list and then summing the result

- mapping the sumEuler function over a list and the summing the result

The main program then adds the two sums to produce the final result. We have chosen arguments which result in a similar run-time for mapFib and mapEuler.

However, when we run this program with one and then two cores we observe no speedup:

satnams@MSRC-LAGAVULIN /papers/afp2008/whnf

$\$$ time WHNF2 +RTS -N1

263935901 


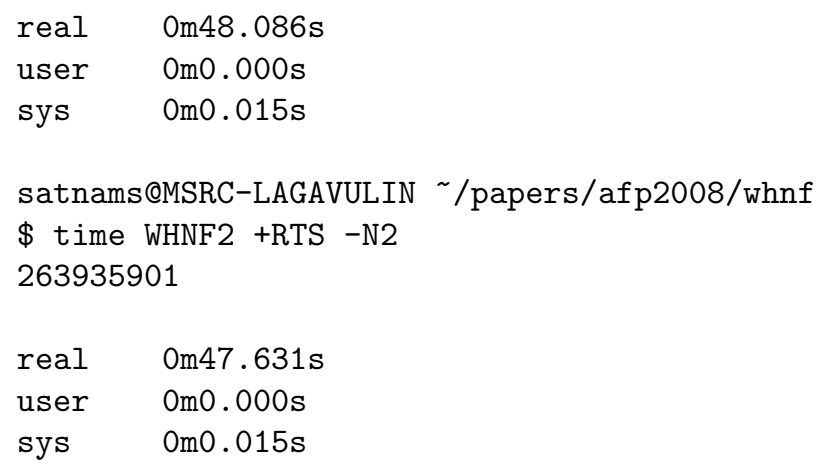

What went wrong? The problem is that the function mapFib does not return a list with four values each fully evaluated to a number. Instead, the expression is reduced to weak head normal form which only return the top level cons cell with the head and the tail elements unevaluated as shown in Figure 8. This means that almost no work is done in the parallel thread. the root of the problem here is Haskell's lazy evaluation strategy which comes into conflict with our desire to control what is evaluated when to help gain performance through parallel execution.

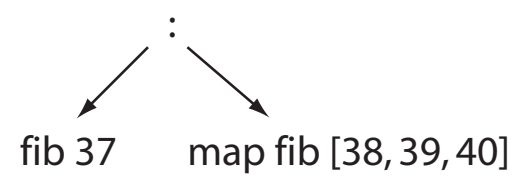

Fig. 8. parFib evaluated to weak head normal form (WHNF)

To fix this problem we need to somehow force the evaluation of the list. We can do this by defining a function that iterates over each element of the list and then uses each element as the first argument to pseq which will cause it to be evaluated to a number:

1 forceList :: [a] $->()$

${ }_{2}$ forcelist [] $=()$

${ }_{3}$ forceList $(\mathrm{x}: \mathrm{xs})=\mathrm{x}$ 'pseq' forceList $\mathrm{xs}$

Using this function we can express our requirement to evaluate the mapFib function fully to a list of numbers rather than to just weak head normal form:

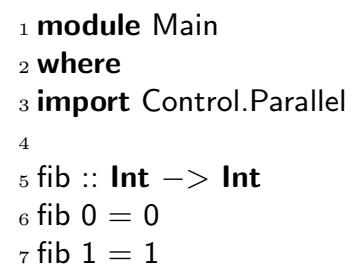




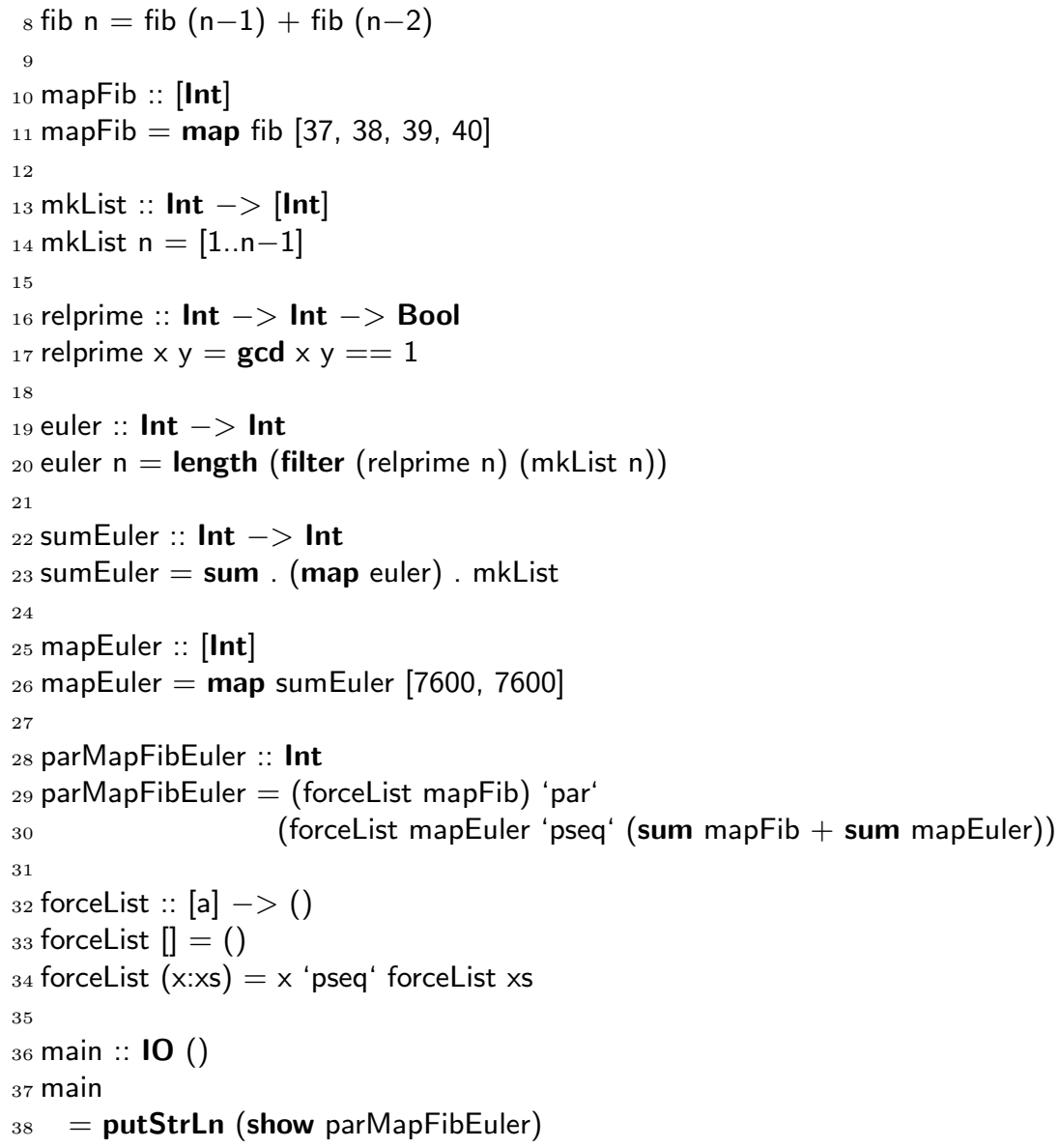

This gives the desired performance which shows the work of mapFib is done in parallel with the work of mapEuler:

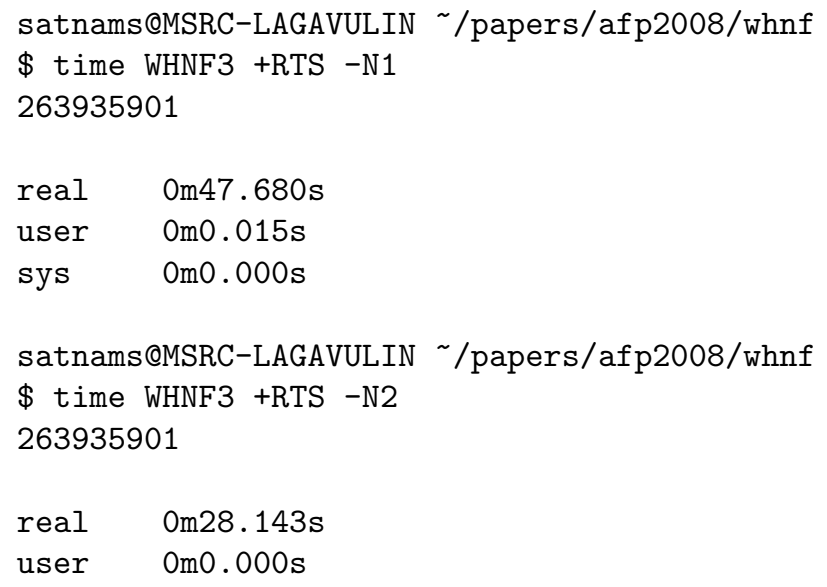


Question. What would be the effect on performance if we omitted the call of forceList on mapEuler?

An important aspect of how pseq works is that it evaluates its first argument to weak head normal formal. This does not fully evaluate an expression e.g. for an expression that constructs a list out of a head and a tail expression (a CONS expression) pseq will not evaluate the head and tail sub-expressions.

Haskell also defines a function called seq but the compiler is free to swap the arguments of seq which means the user can not control evaluation order. The compiler has primitive support for pseq and ensures the arguments are never swapped and this function should always be preferred over seq for parallel programs.

\subsection{Divide and conquer}

Exercise 1: Parallel quicksort. The program below shows a sequential implementation of a quicksort algorithm. Use this program as a template to write a parallel quicksort function. The main body of the program generates a pseudorandom list of numbers and then measures the time taken to build the input list and then to perform the sort and then add up all the numbers in the list.

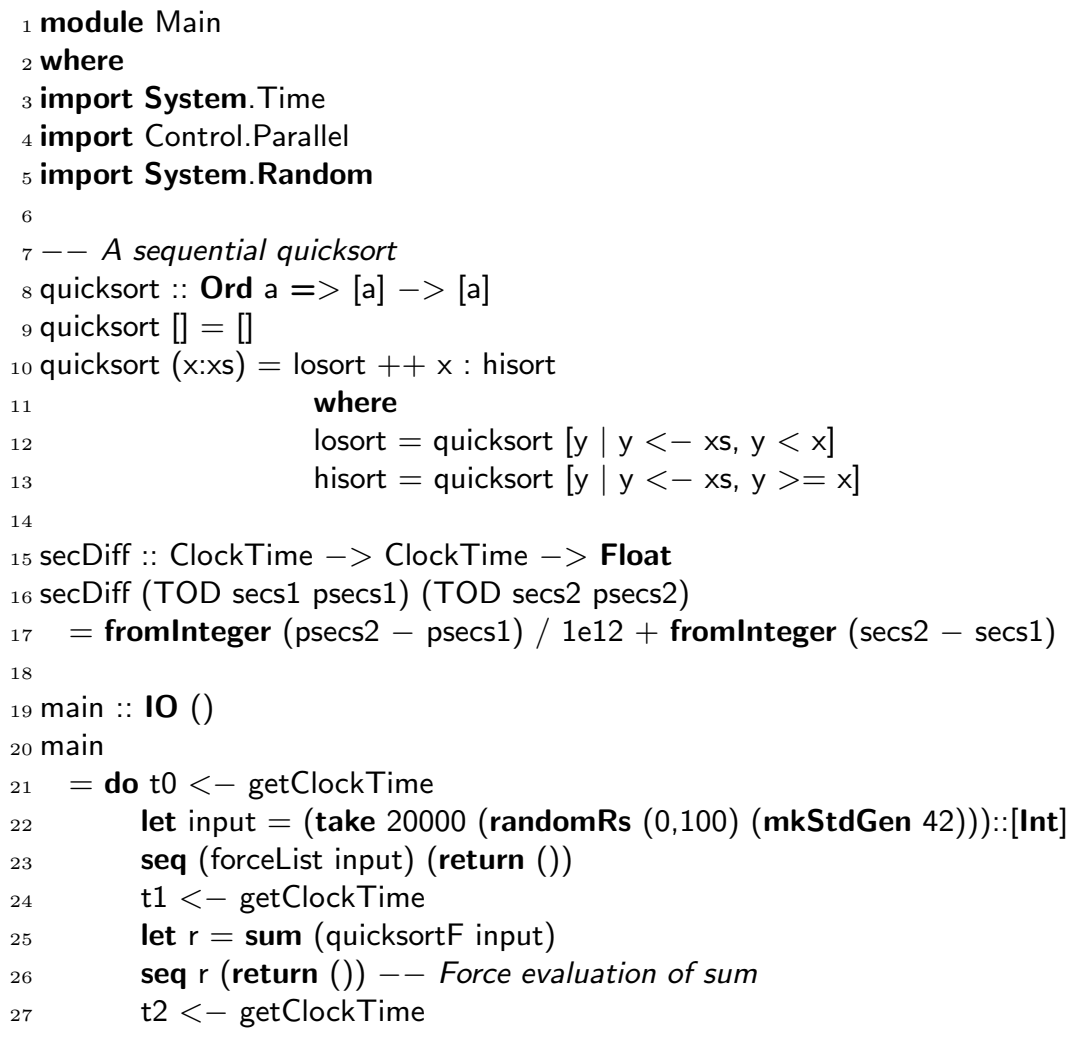


- - Write out the sum of the result.

putStrLn ("Sum of sort: " ++ show r)

- - Write out the time taken to perform the sort.

putStrLn ("Time to sort: " ++ show (secDiff t1 t2))

The performance of a parallel Haskell program can sometimes be improved by reducing the number of garbage collections that occur during execution and a simple way of doing this is to increase the heap size of the program. The size of the heap is specified has an argument to the run-time system e.g. -K100M specifies a 100MB stack and -H800M means use a 800MB heap.

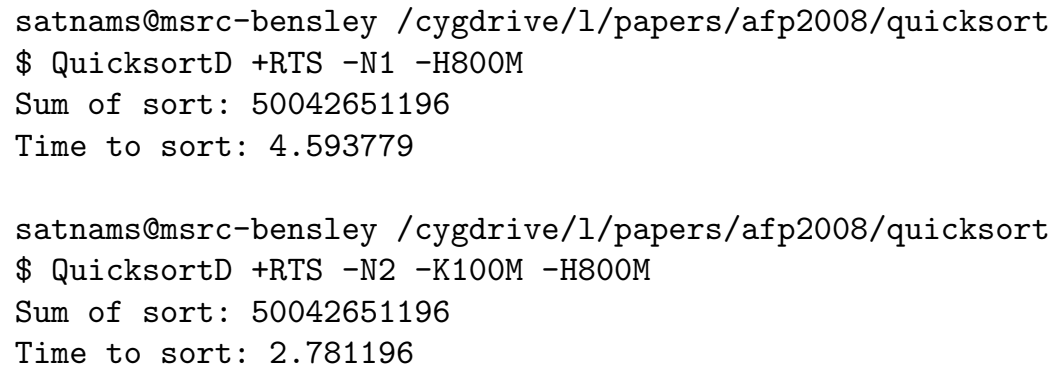

You should consider using par and pseq to try and compute the sub-sorts in parallel. This in itself may not lead to any performance improvement and you should then ensure that the parallel sub-sorts are indeed doing all the work you expect them to do (e.g. consider the effect of lazy evaluation). You may need to write a function to force the evaluation of sub-expressions.

You can get some idea of how well a program has been parallelized and how much time is taken up with garbage collection by using the runtime -s flag to dump some statistics to the standard output. We can also enable GHC's parallel garbage collection and disable load balancing for better cache behaviour with the flags -qg0 -qb.

\$./QuicksortD.exe +RTS -N2 -K100M -H300M -qg0 -qb -s

After execution of a parallel version of quicksort you can look at the end of the file n2.txt to see what happened:

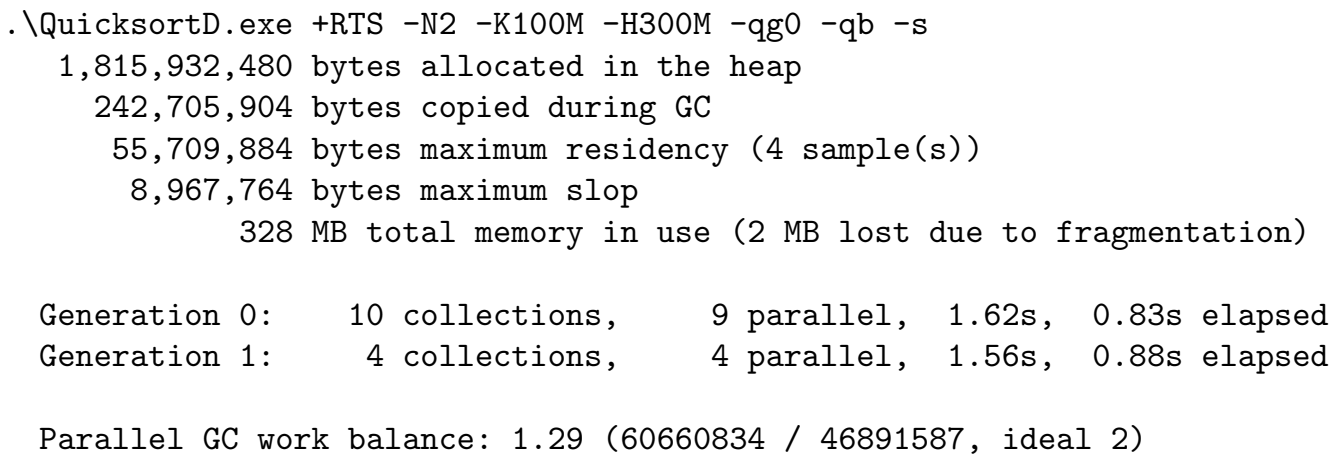




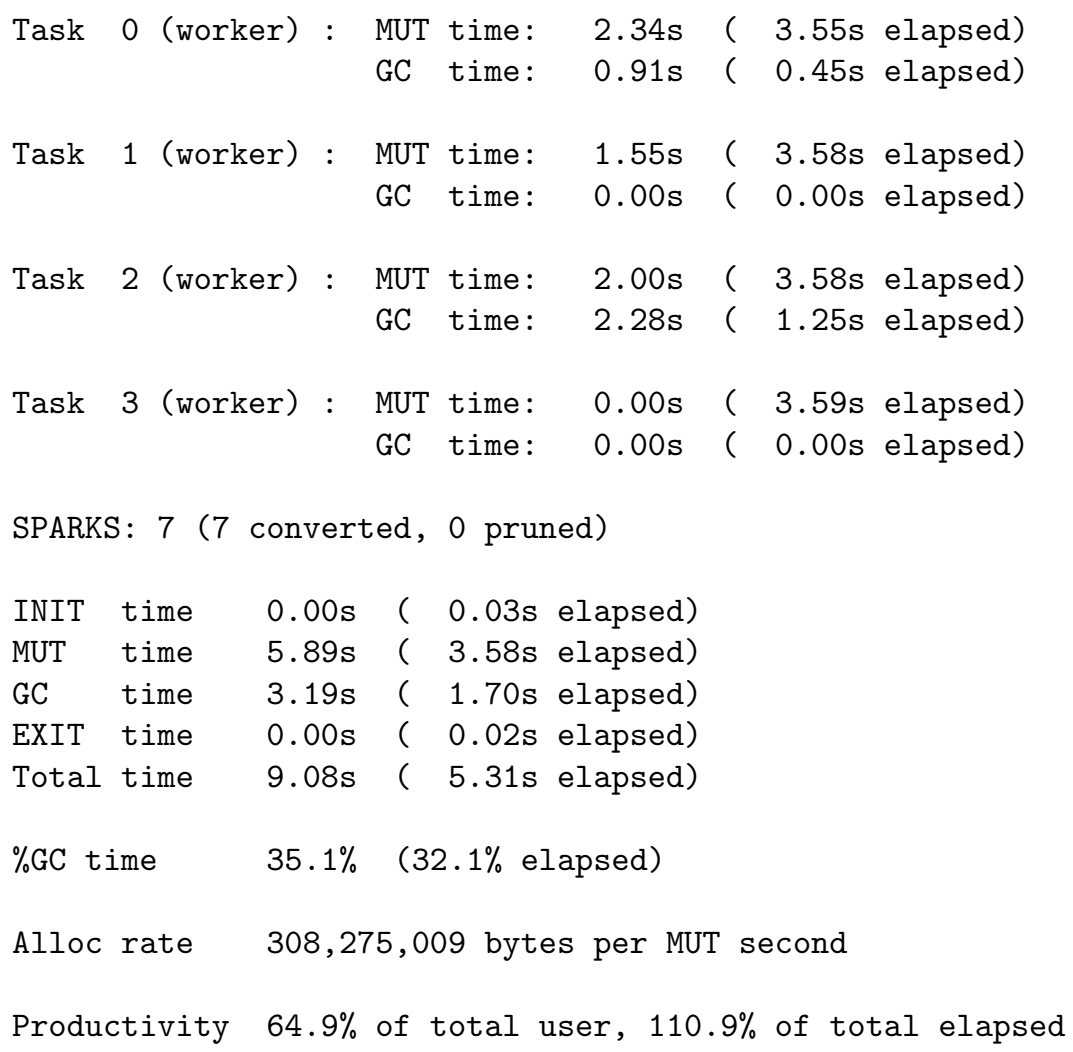

This execution of quicksort spent $35.1 \%$ of its time in garbage collection. The work of the sort was shared out amongst two threads although not evenly. The MUT time gives an indication of how much time was spent performing computation. Seven sparks were created and each of them was evaluated.

\section{Explicit Concurrency}

Writing semi-implicitly parallel programs can sometimes help to parallelize pure functional programs but it does not work when we want to parallelize stateful computations in the IO monad. For that we need to write explicitly threaded programs. In this section we introduce Haskell's mechanisms for writing explicitly concurrent programs. Haskell presents explicit concurrency features to the programmer via a collection of library functions rather than adding special syntactic support for concurrency and all the functions presented in this section are exported by this module.

\subsection{Creating Haskell Threads}

The basic functions for writing explicitly concurrent programs are exported by the Control.Concurrent which defines an abstract type Threadld to allow the identi- 
fication of Haskell threads (which should not be confused with operating system threads). A new thread may be created for any computation in the 10 monad which returns an 10 unit result by calling the forkIO function:

1 forklO :: 10()$->10$ Threadld

Why does the forkIO function take an expression in the 10 monad rather than taking a pure functional expression as its argument? The reason for this is that most concurrent programs need to communicate with each other and this is done through shared synchronized state and these stateful operations have to be carried out in the 10 monad.

One important thing to note about threads that are created by calling forkIO is that the main program (the parent thread) will not automatically wait for the child threads to terminate.

Sometimes it is necessary to use a real operating system thread and this can be achieved using the forkOS function:

1 forkOS :: IO () -> IO Threadld

Threads created by this call are bound to a specific operating system thread and this capability is required to support certain kinds of foreign calls made by Haskell programs to external code.

\subsection{MVars}

To facilitate communication and synchronization between threads Haskell provides MVars ("mutable variables") which can be used to atomically communicate information between threads. MVars and their associated operations are exported by the module Control.Concurrent.MVar. The run-time system ensures that the operations for writing to and reading from MVars occur atomically. An MVar may be empty or it may contain a value. If a thread tries to write to an occupied MVar it is blocked and it will be rewoken when the MVar becomes empty at which point it can try again to atomically write a value into the MVar. If more than one thread is waiting to write to an MVar then the system uses a first-in first-out scheme to wake up just the longest waiting thread. If a thread tries to read from an empty MVar it is blocked and rewoken when a value is written into the MVar when it gets a chance to try and atomically read the new value. Again, if more than one thread is waiting to read from an MVar the run-time system will only wake up the longest waiting thread.

Operations are provided to create an empty MVar, to create a new MVar with an initial value, to remove a value from an MVar, to observe the value in an MVar (plus non-blocking variants) as well as several other useful operations.

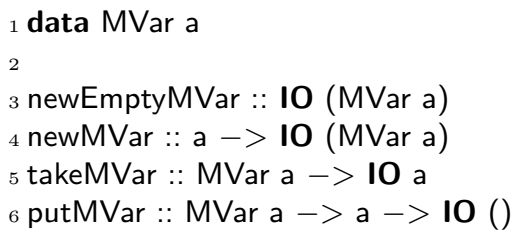




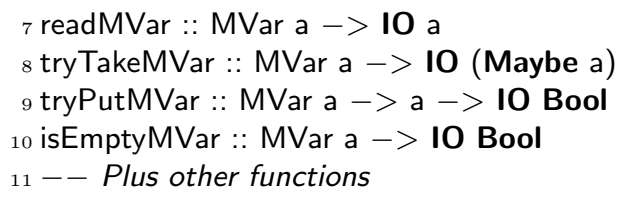

One can use a pair of MVars and the blocking operations putMVar and takeMVar to implement a rendezvous between two threads.

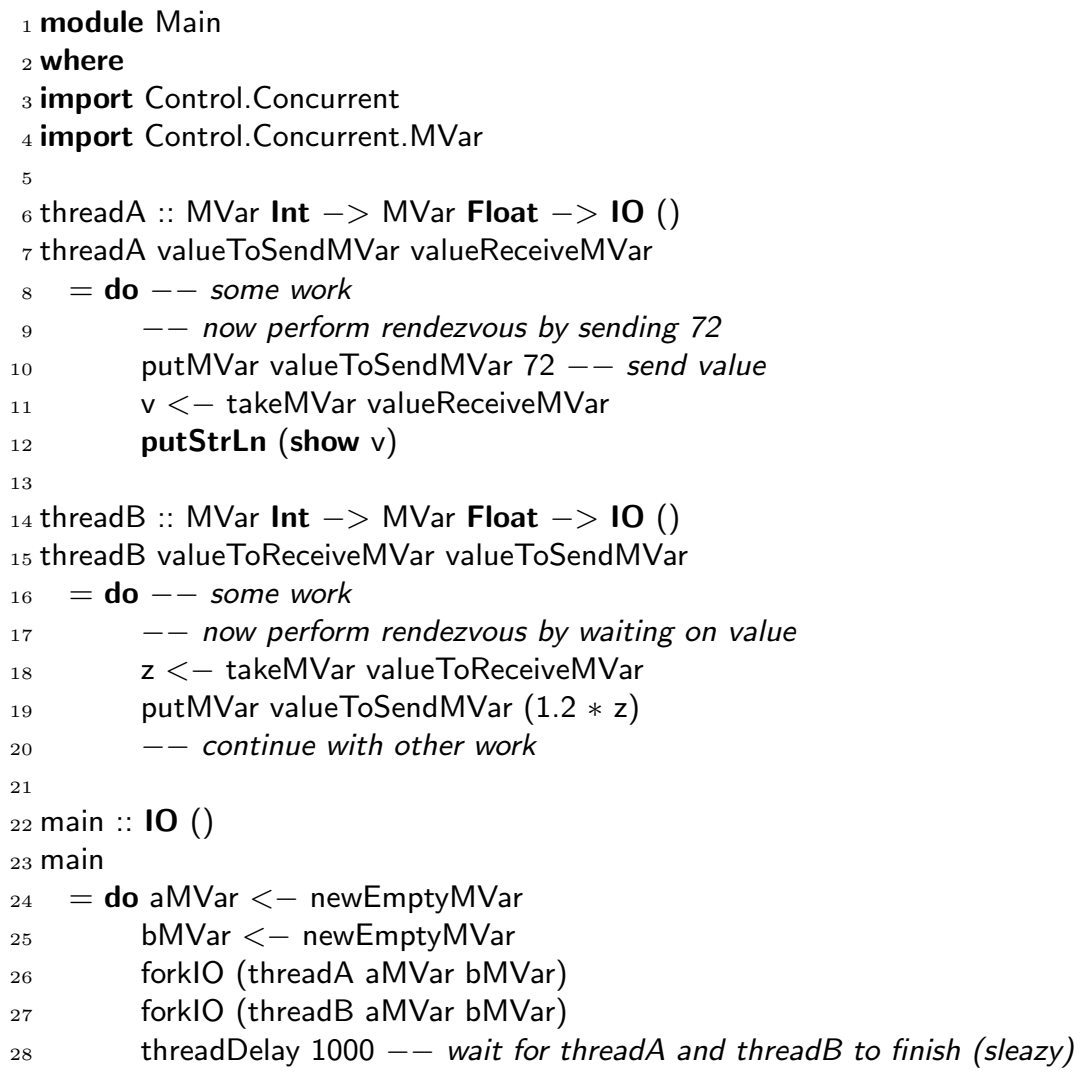

Exercise 2: Re-write this program to remove the use of threadDelay by using some other more robust mechanism to ensure the main thread does not complete until all the child threads have completed.

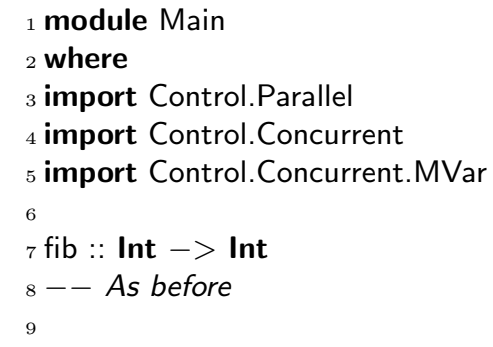




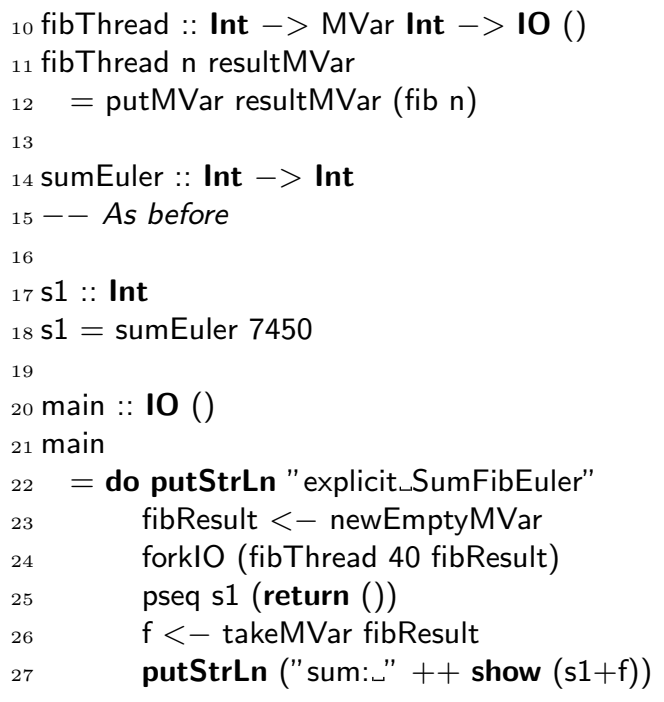

The result of running this program with one and two threads is:

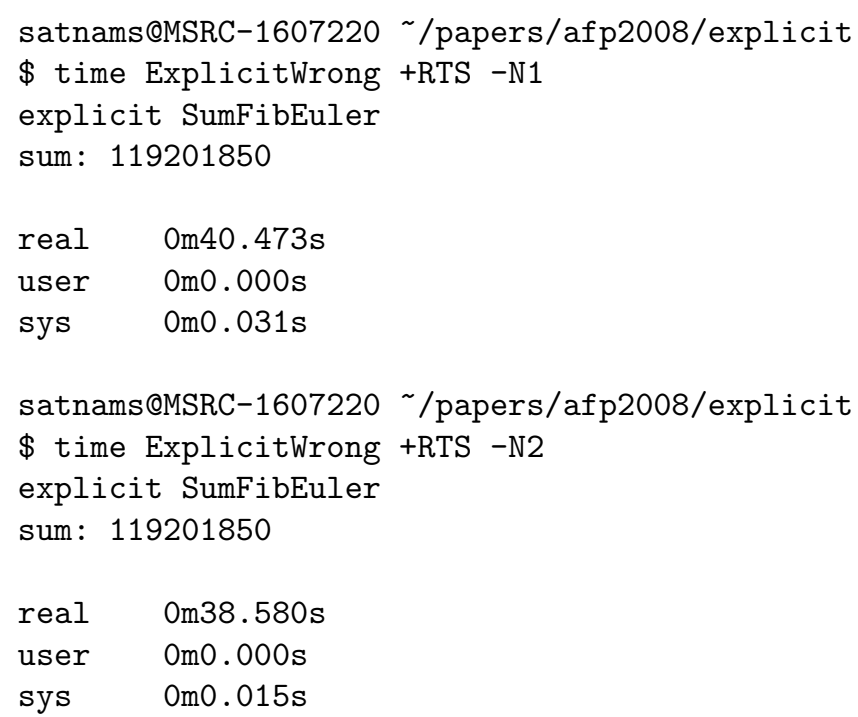

To fix this problem we must ensure the computation of fib fully occurs inside the fibThread thread which we do by using pseq.

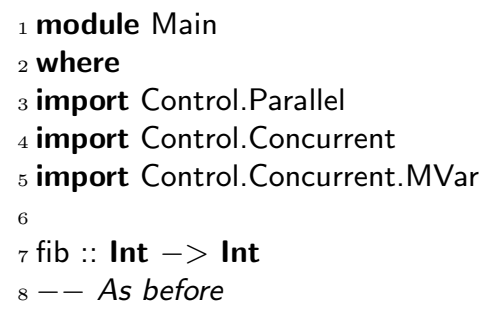




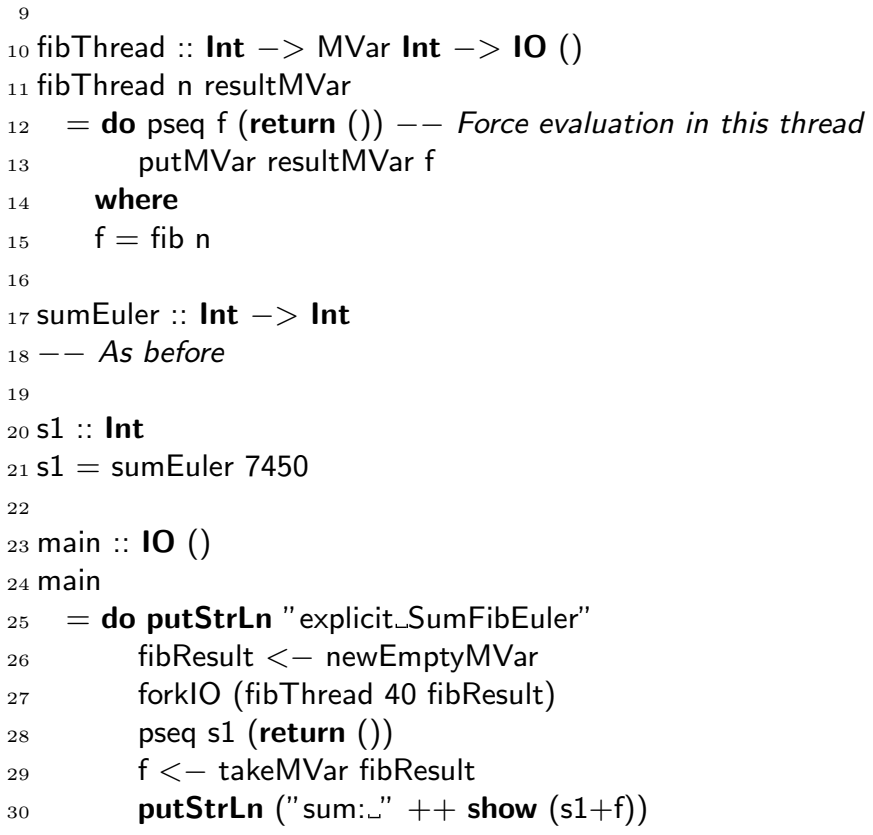

Writing programs with MVars can easily lead to deadlock e.g. when one thread is waiting for a value to appear in an MVar but no other thread will ever write a value into that MVar. Haskell provides an alternative way for threads to synchronize without using explicit locks through the use of software transactional memory (STM) which is accessed via the module Control.Concurrent.STM. A subset of the declarations exposed by this module are shown below.

${ }_{1}$ data STM a - - A monad supporting atomic memory transactions

2 atomically :: STM a $->10$ a -- Perform a series of STM actions atomically

3 retry :: STM a - - Retry current transaction from the beginning

4 orElse :: STM a $->$ STM a $->$ STM a - - Compose two transactions

${ }_{5}$ data TVar a -- Shared memory locations that support atomic memory operations

6 newTVar :: a -> STM (TVar a) - - Create a new TVar with an initial value

7 readTVar :: TVar a $->$ STM a - - Return the current value stored in a TVar

8 writeTVar :: TVar a $->$ a $->$ STM () - - Write the supplied value into a TVar

Software transactional memory works by introducing a special type of shared variable called a TVar rather like a MVar which is used only inside atomic blocks. Inside an atomic block a thread can write and read TVars however outside an atomic block TVars can only be created and passed around around but not read or written. These restrictions are enforced by providing read and write operations that work in the STM monad. The code inside an atomic block is executed as if it were an atomic instruction. One way to think about atomic blocks is to assume that there is one special global lock available and every atomic block works by taking this lock, executing the code in the atomic block, and then releasing this lock. Functionally, it should appear as if no other thread is running in parallel (or no other code is interleaved) with the code in an atomic block. In reality the 
system does allow such parallel and interleaved execution through the use of a $\log$ which is used to roll back the execution of blocks that have conflicting views of shared information.

To execute an atomic block the function atomically takes a computation in the STM monad and executes it in the 10 monad.

To help provide a model for how STM works in Haskell an example is shown in Figures 9 and 10 which illustrates how two threads modify a shared variable using Haskell STM. It is important to note that this is just a model and an actual implementation of STM is much more sophisticated.

Thread 1 tries to atomically increment a shared TVar:

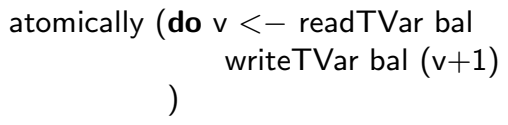

Thread 2 tries to atomically subtract three from a shared TVar:

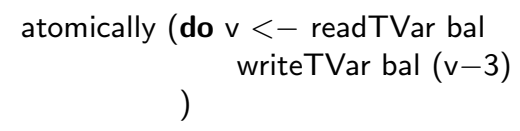

Figure 9(a) shows a shared variable bal with an initial value of 7 and two threads which try to atomically read and update the value of this variable. Thread 1 has an atomic block which atomically increments the value represented by bal. Thread 2 tries to atomically subtract 3 from the value represented by bal. Examples of valid executions include the case where (a) the value represented by bal is first incremented and then has 3 subtracted yielding the value 5 ; or (b) the case where bal has 3 subtracted and then 1 added yielding the value 6 .

Figure 9(b) shows each thread entering its atomic block and a transaction $\log$ is created for each atomic block to record the initial value of the shared variables that are read and to record deferred updates to the shared variable which succeed at commit time if the update is consistent.

Figure 9(c) shows thread 2 reading a value of 7 from the shared variable and this read is recorded its local transaction log.

Figure 9(d) shows that thread 1 also observes a value of 7 from the shared variable which is stored in its transaction log.

Figure 9(e) shows thread 1 updating its view of the shared variable by incrementing it by 1 . This update is made to the local transaction log and not to the shared variable itself. The actual update is deferred until the transaction tries to commit when either it will succeed and the shared variable will be updated or it may fail in which case the log is reset and the transaction is re-run.

Figure $9(\mathrm{f})$ shows thread 2 updating its view of the shared variable to 4 (i.e. 7-3). Now the two threads have inconsistent views of what the value of the shared variable should be.

Figure $9(\mathrm{~g})$ shows thread 1 successfully committing its changes. At commit time the run-time system checks to see if the log contains a consistent value for bal i.e. is the value that has been read in the log the same as the value of the actual bal shared variable? In this case it is i.e. both are 7 so the updated value 8 


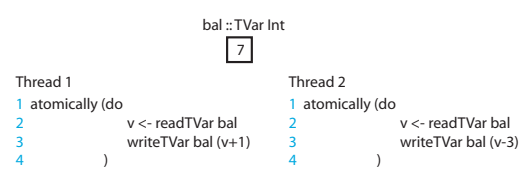

(a) Two threads each with an atomic block and one shared variable
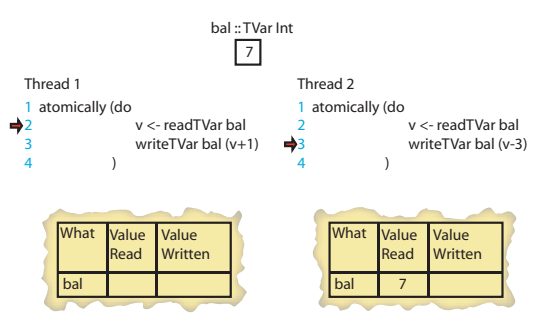

transaction $\log$

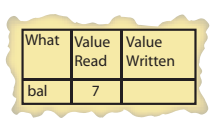

transaction $\log$

(c) Thread 2 reads the value 7 from the shared variable and this read is recorded in its log
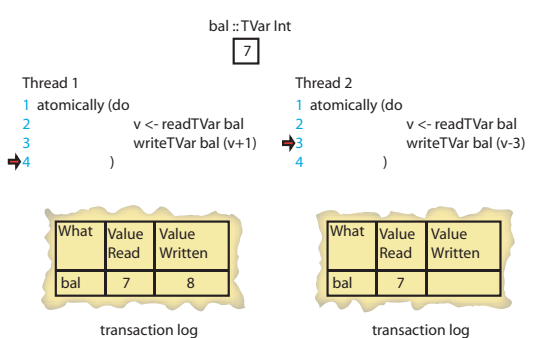

(e) Thread 1 updates its local view of the value of bal to 8 which is put in its own $\log$

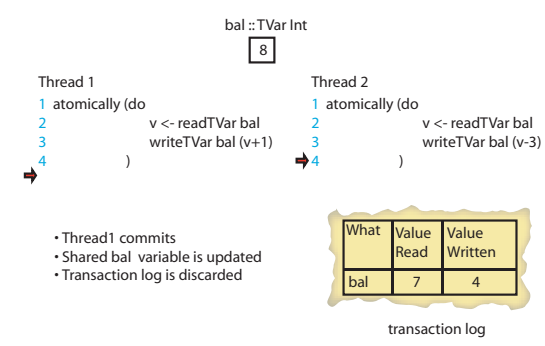

(g) Thread 1 finoshes and updates the shared bal variable and discards its log.

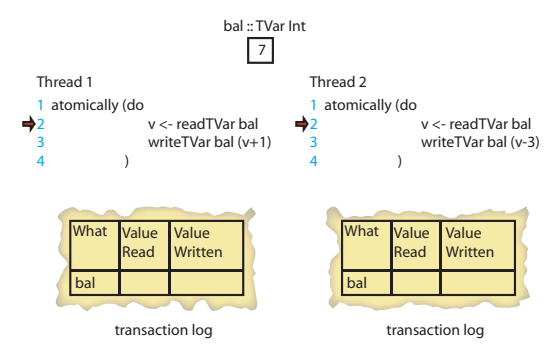

(b) Both threads enter their atomic block and a log is created to track the use of bal
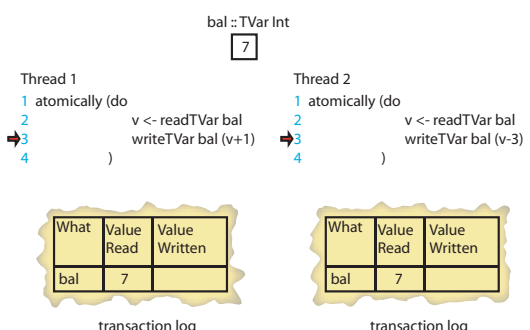

transaction log

(d) Thread 1 also reads the value 7 from the shared variable and this read is recorded in its log

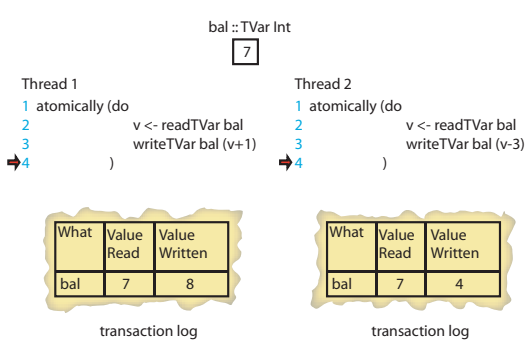

(f) Thread 2 updates its local view of the value of bal to 4 which is put in its own $\log$

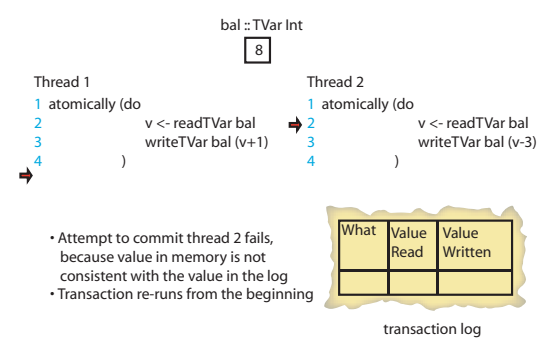

(h) Thread 2 tries to commit its changes which are now inconsistent with the updated value of bal

Fig. 9. A model for STM in Haskell 


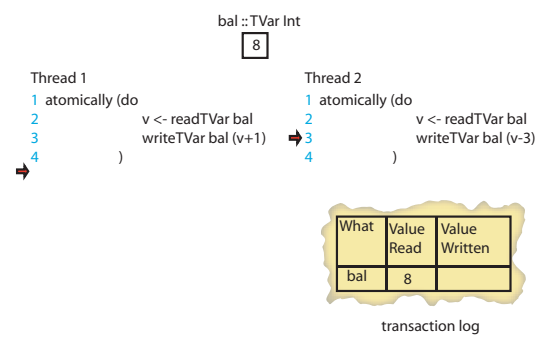

(i) Thread 2 re-executes its atomic block from the start, this time seeing a value of 8 for bal

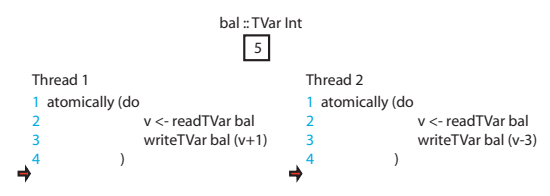

(k) Thread 2 now successfully commits its updates

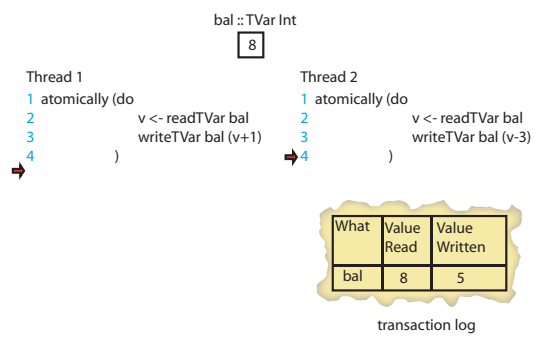

(j) Thread 2 updates it local value for bal

Fig. 10. A model for STM in Haskell (continued)

is written in the shared value. These sequence of events occur atomically. Once the commit succeeds the transaction $\log$ is discarded and the program moves onto the next statement after the atomic block.

Figure 9(h) shows how the commit attempt made by thread 2 fails. This is because thread 2 has recorded a value of 7 for bal but the actual value of bal is 8. This causes the run-time system to erase the values in the log and restart the transaction which will cause it to see the updated value of bal.

Figure 10(i) shows how thread 2 re-executes its atomic block but this time observing the value of 8 for bal.

Figure 10(j) shows thread 2 subtracting 3 from the recorded value of bal to yield an updated value of 5 .

Figure 10(k) shows that thread 2 can now successfully commit with an update of 5 to the shared variable bal. Its transaction $\log$ is discarded.

The retry function allows the code inside an atomic block to abort the current transaction and re-execute it from the beginning using a fresh log. This allows us to implement modular blocking. This is useful when one can determine that a transaction can not commit successfully. The code below shows how a transaction can try to remove money from an account with a case that makes the transaction re-try when there is not enough money in the account. This schedules the atomic block to be run at a later date when hopefully there will be enough money in the account.

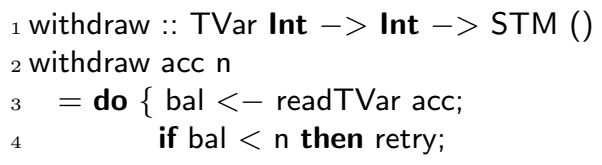




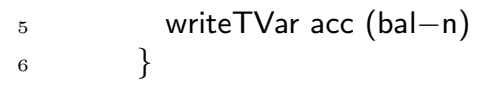

The orElse function allows us to compose two transactions and allows us to implement the notion of choice. If one transaction aborts then the other transaction is executed. If it also aborts then the whole transaction is re-executed. The code below tries to first withdraw money from account a1 and if that fails (i.e. retry is called) it then attempts to withdraw money from account a2 and then it deposits the withdrawn money into account b. If that fails then the whole transaction is re-run.

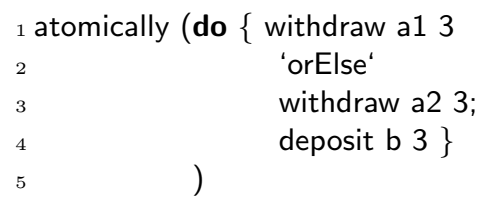

To illustrate the use of software transaction memory we outline how to represent a queue which can be shared between Haskell threads. We shall represent a shared queue with a fixed sized array. A thread that writes to a queue that is full (i.e. the array is full) is blocked and a thread that tries to read from a queue that is empty (i.e. the array is empty) is also blocked. The data-type declaration below for an STM-based queue uses transactional variables to record the head element, the tail element, the empty status and the elements of the array that is used to back to queue.

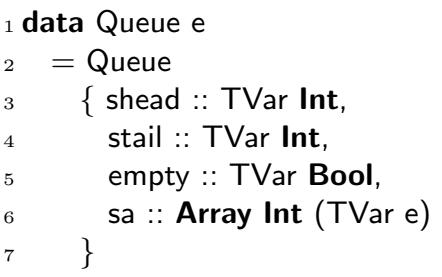

A picture of an empty queue using this representation is shown in Figure 11.

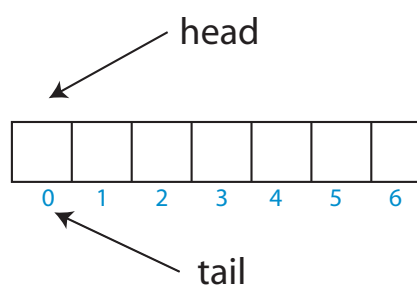

empty:True

Fig. 11. An empty queue

Exercise. Implement the following operations on this STM-based queue representation. 
- Create a new empty queue by defining a function with the type:

i newQueue :: 10 (Queue a)

- Add an element to the queue by defining a function with the type:

enqueue :: Queue a $->$ a $->10()$

If the queue is full the caller should block until space becomes available and the value can be successfully written into the queue.

- Remove an element from the queue and return its value:

dequeue :: Queue a $->10$ a

If the queue is empty the caller should block until there is an item available in the queue for removal.

- Attempt to read a value from a queue and if it is empty then attempt to read a value from a different queue. The caller should block until a value can be obtained from one of the two queues.

dequeueEither :: Queue a $->$ Queue a $->10$ a

\section{$6 \quad$ Nested data parallelism}

This chapter was written in collaboration with Manuel Chakravarty, Gabriele Keller, and Roman Leshchinskiy (University of New South Wales, Sydney).

The two major ways of exploiting parallelism that we have seen so far each have their disadvantages:

- The par/seq style is semantically transparent, but it is hard to ensure that the granularity is consistently large enough to be worth spawning new threads.

- Explicitly-forked threads, communicating using MVars or STM give the programmer precise control over granularity, but at the cost of a new layer of semantic complexity: there are now many threads, each mutating shared memory. Reasoning about all the inter leavings of these threads is hard, especially if there are a lot of them.

Furthermore, neither is easy to implement on a distributed-memory machine, because any pointer can point to any value, so spatial locality is poor. It is possible to support this anarchic memory model on a distributed-memory architecture, as Glasgow Parallel Haskell has shown [3], but it is very hard to get reliable, predictable, and scalable performance. In short, we have no good performance model, which is a Bad Thing if your main purpose in writing a parallel program is to improve performance.

In this chapter we will explore another parallel programming paradigm: data parallelism. The basic idea of data parallelism is simple: 
Do the same thing, in parallel, to every element of a large collection of values.

Not every program can be expressed in this way, but data parallelism is very attractive for those that can, because:

- Everything remains purely functional, like par/seq, so there is no new semantic complexity.

- Granularity is very good: to a first approximation, we get just one thread (with its attendant overheads) for each physical processor, rather than one thread for each data item (of which there are zillions).

- Locality is very good: the data can be physically partitioned across the processors without random cross-heap pointers.

As a result, we get an excellent performance model.

\subsection{Flat data parallelism}

Data parallelism sounds good doesn't it? Indeed, data-parallel programming is widely and successfully used in mainstream languages such as High-Performance Fortran. However, there's a catch: the application has to fit the data-parallel programming paradigm, and only a fairly narrow class of applications do so. But this narrow-ness is largely because mainstream data-parallel technology only supports so-called flat data parallelism. Flat data parallelism works like this

Apply the same sequential function $\mathrm{f}$, in parallel, to every element of a large collection of values a. Not only is $f$ sequential, but it has a similar run-time for each element of the collection.

Here is how we might write such a loop in Data Parallel Haskell:

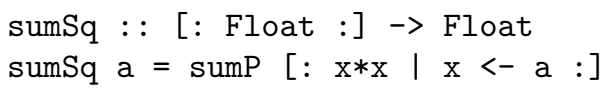

The data type [: Float :] is pronounced "parallel vector of Float". We use a bracket notation reminiscent of lists, because parallel vectors are similar to lists in that consist of an sequence of elements. Many functions available for lists are also available for parallel vectors. For example

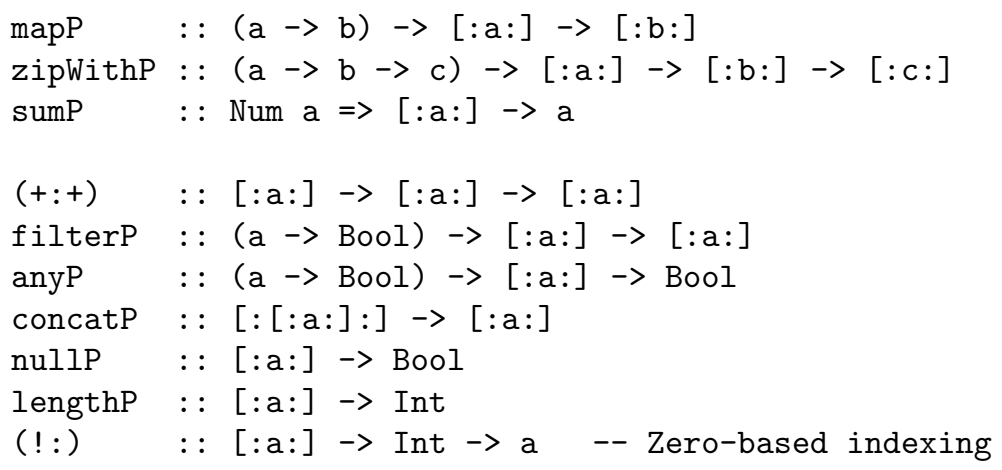


These functions, and many more, are exported by Data.Array.Parallel. Just as we have list comprehensions, we also have parallel-array comprehensions, of which one is used in the above example. But, just as with list comprehensions, array comprehensions are syntactic sugar, and we could just as well have written

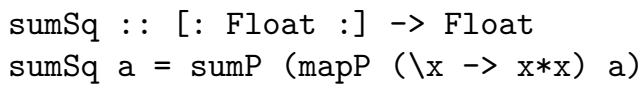

Notice that there is no forkI0, and no par. The parallelism comes implicitly from use of the primitives operating on parallel vectors, such as mapP, sumP, and so on.

Flat data parallelism is not restricted to consuming a single array. For example, here is how we might take the product of two vectors, by multiplying corresponding elements and adding up the results:

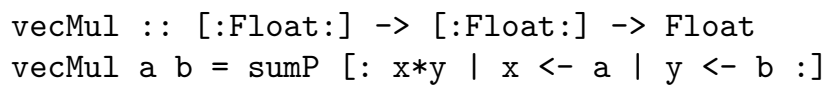

The array comprehension uses a second vertical bar "I" to indicate that we interate over b in lockstep with a. (This same facility is available for ordinary list comprehensions too.) As before the comprehension is just syntactic sugar, and we could have equivalently written this:

vecMul : : [:Float:] $\rightarrow$ [:Float:] $\rightarrow$ Float

vecMul $a b=\operatorname{sumP}(z i p W i t h P(*) a b)$

\subsection{Pros and cons of flat data parallelism}

If you can express your program using flat data parallelism, we can implement it really well on a $\mathrm{N}$-processor machine:

- Divide a into N chunks, one for each processor.

- Compile a sequential loop that applies $f$ successively to each element of a chunk

- Run this loop on each processor

- Combine the results.

Notice that the granularity is good (there is one large-grain thread per processor); locality is good (the elements of a are accessed successively); load-balancing is good (each processor does $1 / N$ of the work). Furthermore the algorithm works well even if $f$ itself does very little work to each element, a situation that is a killer if we spawn a new thread for each invocation of $f$.

In exchange for this great implementation, the programming model is horrible: all the parallelism must come from a single parallel loop. This restriction makes the programming model is very non-compositional. If you have an existing function $g$ written using the data-parallel mapP, you can't call $g$ from another data-parallel map (e.g. mapP g a), because the argument to mapP must be a sequential function. 
Furthermore, just as the control structure must be flat, so must the data structure. We cannot allow a to contain rich nested structure (e.g. the elements of a cannot themselves be vectors), or else similar-run-time promise of $f$ could not be guaranteed, and data locality would be lost.

\subsection{Nested data parallelism}

In the early 90's, Guy Blelloch described nested data-parallel programming. The idea is similar:

Apply the same function $f$, in parallel, to every element of a large collection of values a. However, $f$ may itself be a (nested) data-parallel function, and does not need to have a similar run-time for each element of a.

For example, here is how we might multiply a matrix by a vector:

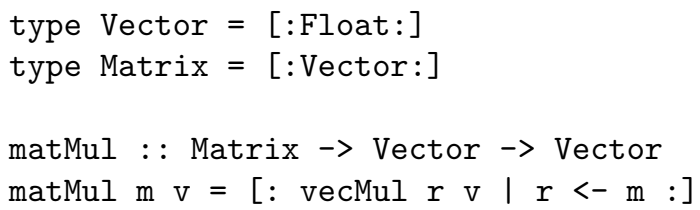

That is, for each row of the matrix, multiply it by the vector $v$ using vecMul. Here we are calling a data-parallel function vecMul from inside a data-parallel operation (the comprehension in matMul).

In very regular examples like this, consisting of visible, nested loops, modern FORTRAN compilers can collapse a loop nest into one loop, and partition the loop across the processors. It is not entirely trivial to do this, but it is well within the reach of compiler technology. But the flattening process only works for the simplest of cases. A typical complication is the matrices may be sparse.

A sparse vector (or matrix) is one in which almost all the elements are zero. We may represent a sparse vector by a (dense) vector of pairs:

type SparseVector $=[:($ Int, Float $):]$

In this representation, only non-zero elements of the vector are represented, by a pair of their index and value. A sparse matrix can now be represented by a (dense) vector of rows, each of which is a sparse vector:

type SparseMatrix $=[$ : SparseVector : $]$

Now we may write vecMul and matMul for sparse arguments thus ${ }^{1}$ :

\footnotetext{
${ }^{1}$ Incidentally, although these functions are very short, they are important in some applications. For example, multiplying a sparse matrix by a dense vector (i.e. sparseMatMul) is the inner loop of the NAS Conjugate Gradient benchmark, consuming $95 \%$ of runtime [4].
} 


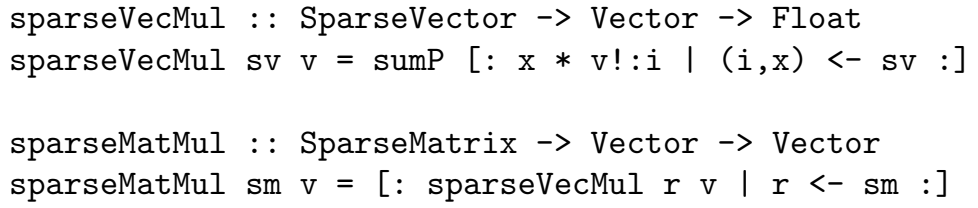

We use the indexing operator (!: ) to index the dense vector v. In this code, the control structure is the same as before (a nested loop, with both levels being data-parallel), but now the data structure is much less regular, and it is much less obvious how to flatten the program into a single data-parallel loop, in such a way that the work is evenly distributed over $\mathrm{N}$ processors, regardless of the distribution of non-zero data in the matrix.

Blelloch's remarkable contribution was to show that it is possible to take any program written using nested data parallelism (easy to write but hard to implement efficiently), and transform it systematically into a program that uses flat data parallelism (hard to write but easy to implement efficiently). He did this for a special-purpose functional language, NESL, designed specifically to demonstrate nested data parallelism.

As a practical programming language, however, NESL is very limited: it is a first-order language, it has only a fixed handful of data types, it is implemented using an interpreter, and so on. Fortunately, in a series of papers, Manuel Chakravarty, Gabriele Keller and Roman Leshchinskiy have generalized Blelloch's transformation to a modern, higher order functional programming language with user-defined algebraic data types - in other words, Haskell. Data Parallel Haskell is a research prototype implementation of all these ideas, in the Glasgow Haskell Compiler, GHC.

The matrix-multiply examples may have suggested to you that Data Parallel Haskell is intended primarily for scientific applications, and that the nesting depth of parallel computations is statically fixed. However the programming paradigm is much more flexible than that. In the rest of this chapter we will give a series of examples of programming in Data Parallel Haskell, designed to help you gain familiarity with the programming style.

Most (in due course, all) of these examples can be found at in the Darcs repos-

itory http://darcs . haskell .org/packages/ndp, in the sub-directory examples/. You can also find a dozen or so other examples of data-parallel algorithms written in NESL at http://www.cs.cmu.edu/ scandal/nesl/algorithms.html.

\subsection{Word search}

Here is a tiny version of a web search engine. A Document is a vector of words, each of which is a string. The task is to find all the occurrences of a word in a large collection of documents, returning the matched documents and the matching word positions in those documents. So here is the type signature for search:

type Document $=[:$ String : $]$ 
type DocColl = [: Document : $]$

search : : DocColl $\rightarrow$ String $\rightarrow$ [: (Document, [:Int:]) :]

We start by solving an easier problem, that of finding all the occurrences of a word in a single document:

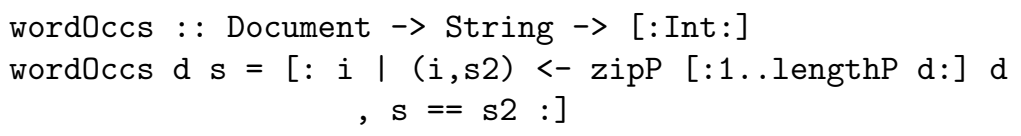

Here we use a filter in the array comprehension, that selects just those pairs ( $i, s 2)$ for which $s==s 2$. Because this is an array comprehension, the implied filtering is performed in data parallel. The ( $i, s 2)$ pairs are chosen from a vector of pairs, itself constructed by zipping the document with the vector of its indices. The latter vector $[: 1 \ldots$ lengthP $\mathrm{d}:]$ is again analogous to the list notation $[1 \ldots \mathrm{n}]$, which generate the list of values between 1 and $\mathrm{n}$. As you can see, in both of these cases (filtering and enumeration) Data Parallel Haskell tries hard to make parallel arrays and vectors as notationally similar as possible.

With this function in hand, it is easy to build search:

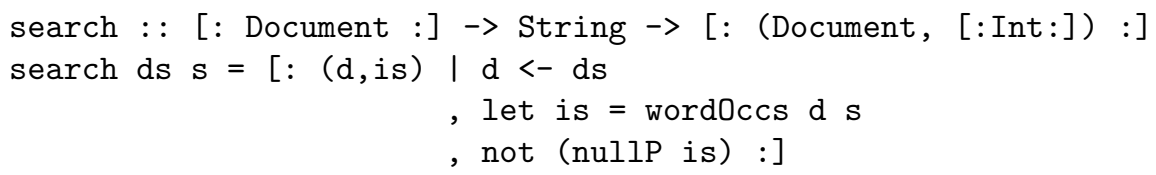

\subsection{Prime numbers}

Let us consider the problem of computing the prime numbers up to a fixed number n, using the sieve of Erathosthenes. You may know the cunning solution using lazy evaluation, thus:

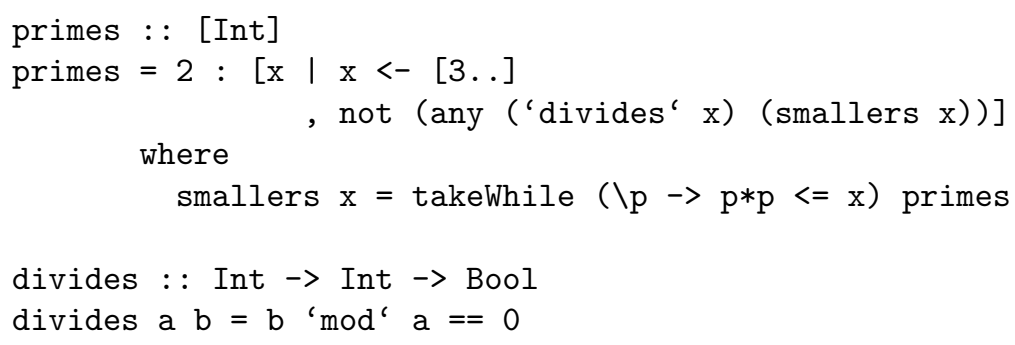

(In fact, this code is not the sieve of Eratosthenes, as Melissa O'Neill's elegant article shows [5], but it will serve our purpose here.) Notice that when considering a candidate prime $\mathrm{x}$, we check that is is not divisible by any prime smaller than the square root of $\mathrm{x}$. This test involves using primes, the very list the definition produces.

How can we do this in parallel? In principle we want to test a whole batch of numbers in parallel for prime factors. So we must specify how big the batch is: 


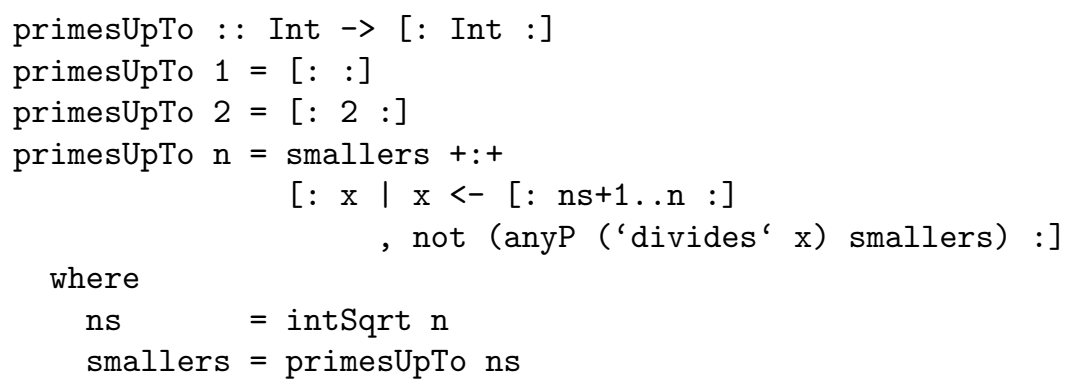

As in the case of word0ccs, we use a boolean condition in a comprehension to filter the candidate primes. This time, however, computing the condition itself is a nested data-parallel computation (as it was in search). used here to filter candidate primes $\mathrm{x}$.

To compute smallers we make a recursive call to primesUpTo. This makes primesUpTo unlike all the previous examples: the depth of data-parallel nesting is determined dynamically, rather than being statically fixed to depth two. It should be clear that the structure of the parallelism is now much more complicated than before, and well out of the reach of mainstream flat data-parallel systems. But it has abundant data parallelism, and will execute with scalable performance on a parallel processor.

\subsection{Quicksort}

In all the examples so far the "branching factor" has been large. That is, each data-parallel operations has worked on a large collection. What happens if the collection is much smaller? For example, a divide-and-conquer algorithm usually divides a problem into a handful (perhaps only two) sub-problems, solves them, and combines the results. If we visualize the tree of tasks for a divide-and-conquer algorithm, it will have a small branching factor at each node, and may be highly un-balanced.

Is this amenable to nested data parallelism? Yes, it is. Quicksort is a classic divide-and-conquer algorithm, and one that we have already studied. Here it is, expressed in Data Parallel Haskell:

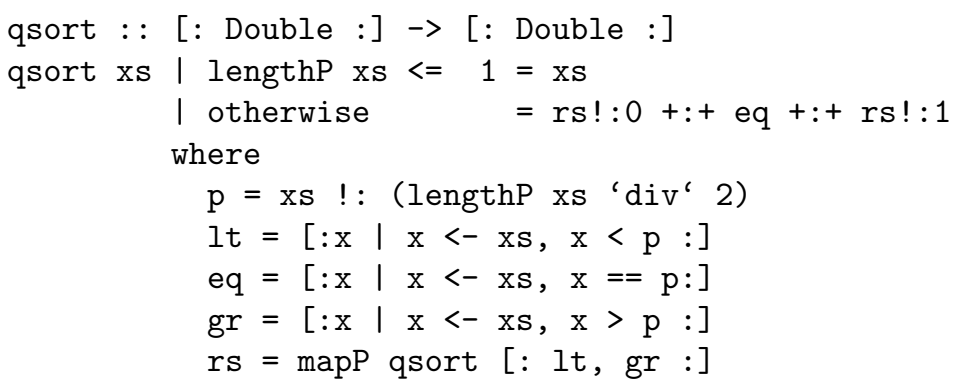

The crucial step here is the use of mapP on a two-element array [: lt, gr :]. This says "in data-parallel, apply qsort to lt and gr". The fact that there are 
only two elements in the vector does not matter. If you visualize the binary tree of sorting tasks that quicksort generates, then each horizontal layer of the tree is done in data-parallel, even though each layer consists of many unrelated sorting tasks.

\subsection{Barnes Hut}

All our previous examples worked on simple flat or nested collections. Let's now have a look at an algorithm based on a more complex structure, in which the elements of a parallel array come from a recursive and user-defined algebraic data type.

In the following, we present an implementation ${ }^{2}$ of a simple version of the Barnes-Hut $n$-body algorithm[7], which is a representative of an important class of parallel algorithms covering applications like simulation and radiocity computations. These algorithms consist of two main steps: first, the data is clustered in a hierarchical tree structure; then, the data is traversed according to the hierarchical structure computed in the first step. In general, we have the situation that the computations that have to be applied to data on the same level of the tree can be executed in parallel. Let us first have a look at the Barnes-Hut algorithm and the data structures that are required, before we discuss the actual implementation in parallel Haskell.

An $n$-body algorithm determines the interaction between a set of particles by computing the forces which act between each pair of particles. A precise solution therefore requires the computations of $n^{2}$ forces, which is not feasible for large numbers of particles. The Barnes-Hut algorithm minimizes the number of force calculations by grouping particles hierarchically into cells according to their spatial position. The hierarchy is represented by a tree. This allows approximating the accelerations induced by a group of particles on distant particles by using the centroid of that group's cell. The algorithm has two phases: (1) The tree is constructed from a particle set, and (2) the acceleration for each particle is computed in a down-sweep over the tree. Each particle is represented by a value of type MassPoint, a pair of position in the two dimensional space and mass:

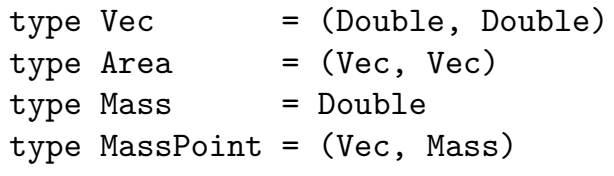

We represent the tree as a node which contains the centroid and a parallel array of subtrees:

data Tree $=$ Node MassPoint [:Tree:]

Notice that a Tree contains a parallel array of Tree.

Each iteration of bhTree takes the current particle set and the area in which the particles are located as parameters. It first splits the area into four subareas

\footnotetext{
${ }^{2}$ Our description here is based heavily on that in [6].
} 


\begin{tabular}{|c|c|c|c|c|}
\hline & & & \multirow{2}{*}{\multicolumn{2}{|c|}{$p_{1}$}} \\
\hline & \multicolumn{2}{|c|}{$p_{2}$} & & \\
\hline & $p_{7}$ & $p_{6}$ & $p_{4}$ & \\
\hline$p_{8}$ & & & & \multirow{2}{*}{$p_{5}$} \\
\hline$p_{9}$ & & & & \\
\hline
\end{tabular}

Fig. 12. Hierarchical division of an area into subareas

subAs of equal size. It then subdivides the particles into four subsets according to the subarea they are located in. Then, bhTree is called recursively for each subset and subarea. The resulting four trees are the subtrees of the tree representing the particles of the area, and the centroid of their roots is the centroid of the complete area. Once an area contains only one particle, the recursion terminates. Figure 12 shows such a decomposition of an area for a given set of particles, and Figure 13 displays the resulting tree structure.

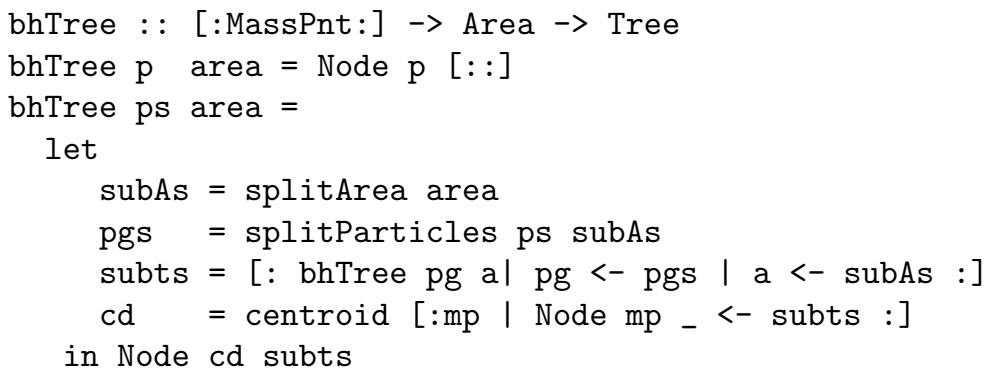

The tree computed by bhTree is then used to compute the forces that act on each particle by a function accels. It first splits the set of particles into two subsets: fMps, which contains the particles far away (according to a given criteria), and cMps, which contains those close to the centroid stored in the root of the tree. For all particles in fMps, the acceleration is approximated by computing the interaction between the particle and the centroid. Then, accels is called recursively for with cMps and each of the subtrees. The computation terminates once there are no particles left in the set.

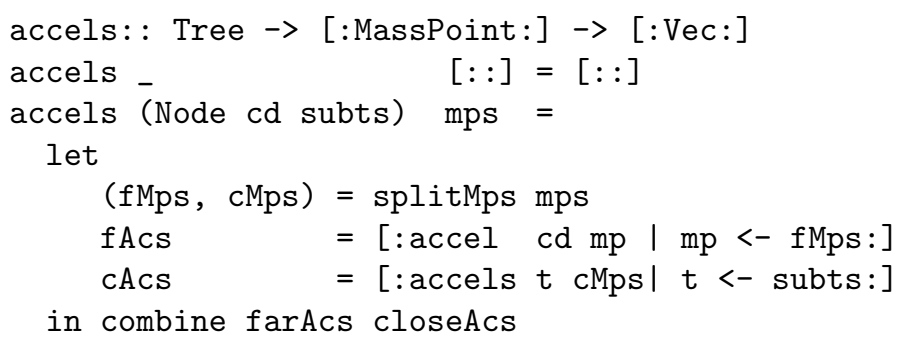




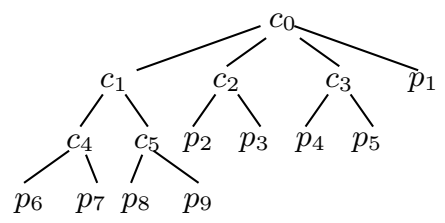

Fig. 13. Example of a Barnes-Hut tree.

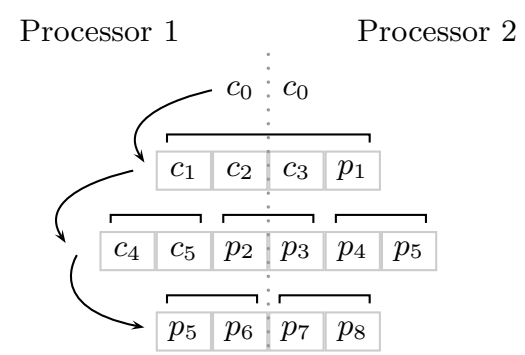

Fig. 14. Distribution of the values of the flattened tree

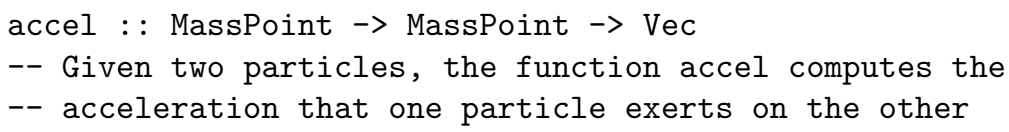

The tree is both built and traversed level by level, i.e., all nodes in one level of the tree are processed in a single parallel step, one level after the other. This information is important for the compiler to achieve good data locality and load balance, because it implies that each processor should have approximately the same number of masspoints of each level. We can see the tree as having a sequential dimension to it, its depth, and a parallel dimension, the breadth, neither of which can be predicted statically. The programmer conveys this information to the compiler by the choice the data structure: By putting all subtrees into a parallel array in the type definition, the compiler assumes that all subtrees are going to be processed in parallel. The depth of the tree is modeled by the recursion in the type, which is inherently sequential.

\subsection{A performance model}

One of the main advantages of the data parallel programming model is that it comes with a performance model that lets us make reasonable predictions about the behavior of the program on a parallel machine, including its scalability - that is, how performance changes as we add processors. So what is this performance model? 
First, we must make explicit something we have glossed over thus far: dataparallel arrays are strict. More precisely, if any element of a parallel array diverges, then all elements diverge ${ }^{3}$. This makes sense, because if we demand any element of a parallel array then we must compute them all in data parallel; and if that computation diverges we are justified in not returning any of them. The same constraint means that we can represent parallel arrays very efficiently. For example, an array of floats, [:Float:], is represented by a contiguous array of unboxed floating-point numbers. There are no pointers, and iterating over the array has excellent spatial locality.

In reasoning about performance, Blelloch [9] characterizes the work and depth of the program:

- The work, $W$, of the program is the time it would take to execute on a single processor.

- The depth, $D$, of the program is the time it would take to execute on an infinite number processors, under the assumption that the additional processors leap into action when (but only when) a mapP, or other data-parallel primitive, is executed.

If you think of the unrolled data-flow diagram for the program, the work is the number of nodes in the data-flow diagram, while the depth is the longest path from input to output.

Of course, we do not have an infinite number of processors. Suppose instead that we have $P$ processors. Then if everything worked perfectly, the work be precisely evenly balanced across the processors and the execution time $T$ would be $W / P$. That will not happen if the depth $D$ is very large. So in fact, we have

$$
W / P \leq T \leq W / P+L * D
$$

where $L$ is a constant that grows with the latency of communication in the machine. Even this is a wild approximation, because it takes no account of bandwidth limitations. For example, between each of the recursive calls in the Quicksort example there must be some data movement to bring together the elements less than, equal to, and greater than the pivot. Nevertheless, if the network bandwidth of the parallel machine is high (and on serious multiprocessors it usually is) the model gives a reasonable approximation.

How can we compute work and depth? It is much easier to reason about the work of a program in a strict setting than in a lazy one, because all subexpressions are evaluated. This is why the performance model of the data-parallel part of DPH is more tractable than for Haskell itself.

The computation of depth is where we take account of data parallelism. Figure 15 shows the equations for calculating the depth of a closed expression $e$, where $\mathcal{D} \llbracket e \rrbracket$ means "the depth of $e$ ". These equations embody the following ideas:

- By default execution is sequential. Hence, the depth of an addition is the sum of the depths of its arguments.

\footnotetext{
${ }^{3}$ What if the elements are pairs? See Leshchinskiy's thesis for the details [8].
} 


$$
\begin{aligned}
& \mathcal{D} \llbracket k \rrbracket=0 \\
& \mathcal{D} \llbracket x \rrbracket=0 \\
& \mathcal{D} \llbracket e_{1}+e_{2} \rrbracket=1+\mathcal{D} \llbracket e_{1} \rrbracket+\mathcal{D} \llbracket e_{2} \rrbracket \\
& \mathcal{D} \llbracket \text { if } e_{1} \text { then } e_{2} \text { else } e_{3} \rrbracket=\mathcal{D} \llbracket e_{1} \rrbracket+\mathcal{D} \llbracket e_{2} \rrbracket \\
& =\mathcal{D} \llbracket e_{1} \rrbracket+\mathcal{D} \llbracket e_{3} \rrbracket \\
& \mathcal{D} \llbracket \text { let } x=e \text { in } b \rrbracket=\mathcal{D} \llbracket b[e / x] \rrbracket \\
& \mathcal{D} \llbracket e_{1}+:+e_{2} \rrbracket=1+\mathcal{D} \llbracket e_{1} \rrbracket+\mathcal{D} \llbracket e_{2} \rrbracket \\
& \mathcal{D} \llbracket \text { concatP } e \rrbracket=1+\mathcal{D} \llbracket e \rrbracket \\
& \mathcal{D} \llbracket \operatorname{map} P \quad f e \rrbracket=1+\mathcal{D} \llbracket e \rrbracket+\max _{x \in e} \mathcal{D} \llbracket f x \rrbracket \\
& \mathcal{D} \llbracket \text { filterP } f e \rrbracket=1+\mathcal{D} \llbracket e \rrbracket+\mathcal{D} \llbracket f \rrbracket \\
& \mathcal{D} \llbracket \operatorname{sumP} e \rrbracket=1+\mathcal{D} \llbracket e \rrbracket+\log (\text { length }(e))
\end{aligned}
$$

Fig. 15. Depth model for closed expressions

- The parallel primitive mapP, and its relatives such as filterP, can take advantage of parallelism, so the depth is the worst depth encountered for any element.

- The parallel reduction primitive sumP, and its relatives, take time logarithmic in the length of the array.

The rule for mapP dirctly embodies the idea that nested data parallelism is flattened. For example, suppose $e::[:[:$ Float: $]:]$. Then, applying the rules we see that

$$
\begin{aligned}
\mathcal{D} \llbracket \text { mapP } f \text { (concatP } e \rrbracket & =1+\mathcal{D} \llbracket \operatorname{concatP} e \rrbracket+\max _{x \in \operatorname{concatP} e} \mathcal{D} \llbracket f x \rrbracket \\
& =1+1+\mathcal{D} \llbracket e \rrbracket+\max _{x \in \operatorname{concatP} e} \mathcal{D} \llbracket f x \rrbracket \\
& =2+\mathcal{D} \llbracket e \rrbracket+\max _{x s \in e} \max _{x \in x s} \mathcal{D} \llbracket f x \rrbracket \\
\mathcal{D} \llbracket \operatorname{mapP}(\operatorname{mapP} f) e \rrbracket & =1+\mathcal{D} \llbracket e \rrbracket+\max _{x s \in e} \mathcal{D} \llbracket \operatorname{mapP} f x s \rrbracket \\
& =1+\mathcal{D} \llbracket e \rrbracket+1+\max _{x s \in e} \max _{x \in x s} \mathcal{D} \llbracket f x \rrbracket \\
& =2+\mathcal{D} \llbracket e \rrbracket+\max _{x s \in e} \max _{x \in x s} \mathcal{D} \llbracket f x \rrbracket
\end{aligned}
$$

Notice that although the second case is a nested data-parallel computation, it has the same depth expression as the first: the data-parallel nesting is flattened.

These calculations are obviously very approximate, certainly so far as constant factors are concerned. For example, in the inequality for execution time,

$$
W / P \leq T \leq W / P+L * D
$$

we do not know the value of the latency-related constant $L$. However, what we are primarily looking for is the Asymptotic Scalability (AS) property: 
A program has the Asymptotic Scalability property if $D$ grows asymptotically more slowly than $W$, as the size of the problem increases.

If this is so then, for a sufficiently large problem and assuming sufficient network bandwidth, performance should scale linearly with the number of processors.

For example, the functions sumSq and search both have constant depth, so both have the AS property, and (assuming sufficient bandwidth) performance should scale linearly with the number of processors after some fairly low threshold.

For Quicksort, an inductive argument shows that the depth is logarithmic in the size of the array, assuming the pivot is not badly chosen. So $W=O(n \log n)$ and $D=O(\log n)$, and Quicksort has the AS property.

For computing primes, the depth is smaller: $D=O(\log \log n)$. Why? Because at every step we take the square root of $n$, so that at depth $d$ we have $n=2^{2^{d}}$. Almost all the work is done at the top level. The work at each level involves comparing all the numbers between $\sqrt{n}$ and $n$ with each prime smaller than $\sqrt{n}$. There are approximately $\sqrt{n} / \log n$ primes smaller than $\sqrt{n}$, so the total work is roughly $W=O\left(n^{3 / 2} / \log n\right)$. So again we have the AS property.

Leshchinskiy et al [10] give further details of the cost model.

\subsection{How it works}

NESL's key insight is that it is possible to transform a program that uses nested data-parallelism into one that uses only flat data parallelism. While this little miracle happens behind the scenes, it is instructive to have some idea how it works, just as a car driver may find some knowledge of internal combustion engines even if he is not a skilled mechanic. The description here is necessarily brief, but the reader may find a slightly more detailed overview in [11], and in the papers cited there.

We call the nested-to-flat transformation the vectorization transform. It has two parts:

- Transform the data so that all parallel arrays contain only primitive, flat data, such as Int, Float, Double.

- Transform the code to manipulate this flat data.

To begin with, let us focus on the first of these topics. We may consider it as the driving force, because nesting of data-parallel operations is often driven by nested data structures.

Transforming the data As we have already discussed, a parallel array of Float is represented by a contiguous array of honest-to-goodness IEEE floating point numbers; and similarly for Int and Double. It is as if we could define the parallel-array type by cases, thus:

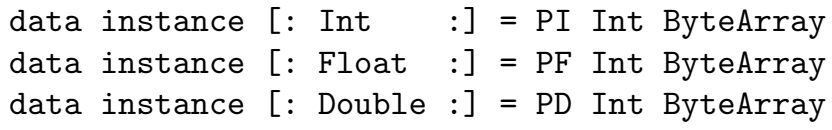


In each case the Int field is the size of the array. These data declarations are unusual because they are non-parametric: the representation of an array depends on the type of the elements ${ }^{4}$.

Matters become even more interesting when we want to represent a parallel array of pairs. We must not represent it as a vector of pointers to heap-allocated pairs, scattered randomly around the address space. We get much better locality if we instead represent it as a pair of arrays thus:

$$
\text { data instance }[:(\mathrm{a}, \mathrm{b}):]=\operatorname{PP}[: \mathrm{a}:] \quad[: \mathrm{b}:]
$$

Note that elements of vectors are hyperstrict. What about a parallel array of parallel arrays? Again, we must avoid a vector of pointers. Instead, the natural representation is obtained by literally concatenating the (representation of) the sub-vectors into one giant vector, together with a vector of indices to indicate where each of the sub-vectors begins.

data instance $[:[: \mathrm{a}:]:]=\mathrm{PA}[:$ Int $:][: \mathrm{a}:]$

By way of example, recall the data types for sparse matrices:

type SparseMatrix $=[:$ SparseVector : $]$

type SparseVector $=[$ : (Int, Float) : $]$

Now consider this tiny matrix, consisting of two short documents:

$\mathrm{m}:$ : SparseMatrix

$\mathrm{m}=[:[:(1,2.0),(7,1.9):],[:(3,3.0):]:]$

This would be represented as follows:

$$
\begin{aligned}
\mathrm{PA}[: 0,2:] \quad(\mathrm{PP} \quad & {[: 1,7,3: 3] } \\
& {[: 1.0,1.9,3.0:]) }
\end{aligned}
$$

The array (just like the leaves) are themselves represented as byte arrays:

$$
\begin{aligned}
& \text { PA (PI } 2 \#<0 \times 0,0 \times 2>\text { ) } \\
& \text { (PP (PI } 3 \#<0 \times 1, \quad 0 \times 7, \quad 0 \times 3>) \\
& \text { (PF } 3 \text { \#<0x9383, 0x92818, 0x91813>)) }
\end{aligned}
$$

Here we have invented a fanciful notation for literal ByteArrays (not supported by GHC, let alone Haskell) to stress the fact that in the end everything boils down to literal bytes. (The hexadecimal encodings of floating point numbers are also made up because the real ones have many digits!)

We have not discussed how to represent arrays of sum types (such as Bool, Maybe, or lists), nor of function types - see [14] and [8] respectively.

\footnotetext{
${ }^{4}$ None of this is visible to the programmer, but the data instance notation is in fact available to the programmer in recent versions of GHC $[12,13]$. Why? Because GHC has a typed intermediate language so we needed to figure out how to give a typed account of the vectorization transformation, and once that is done it seems natural to offer it to the programmer. Furthermore, much of the low-level support code for nested data parallelism is itself written in Haskell, and operates directly on the post-vectorization array representation.
} 
Vectorising the code As you can see, data structures are transformed quite radically by the vectorisation transform, and it follows that the code must be equally radically transformed. Space precludes proper treatment here; a good starting point is Keller's thesis [15].

A data-parallel program has many array-valued sub-expressions. For example, in sumSq we see

sumSq $\mathrm{a}=\operatorname{sum} P[: \mathrm{x} * \mathrm{x} \mid \mathrm{x}<-\mathrm{a}:]$

However, if a is a big array, it would be silly to compute a new, equally big array of squares, only to immediately consume it with sump. It would be much better for each processor to zip down its chunk of a, adding the square of each element into a running total, and for each processor's total to be combined.

The elimination of intermediate arrays is called fusion and is crucial to improve the constant factor of Data Parallel Haskell. It turns out that vectorisation introduces many more intermediate arrays, which makes fusion even more important. These constant factors are extremely important in practice: if there is a slow-down of a factor of 50 relative to $\mathrm{C}$, then even if you get linear speedup by adding processors, Data Parallel Haskell is unlikely to become popular.

\subsection{Running Data Parallel Haskell}

GHC 6.6 and 6.8 come with support for Data Parallel Haskell syntax, and a purely sequential implementation of the operations. So you can readily try out all of the examples in this paper, and ones of your own devising thus:

- Use ghc or ghci version 6.6.x or 6.8.x.

- Use flags -fparr and -XParallelListComp.

- Import module GHC. PArr.

Some support for genuinely-parallel Data Parallel Haskell, including the allimportant vectorisation transformation, will be in GHC 6.10 (planned release: autumn 2008). It is not yet clear just how complete the support will be at that time. At the time of writing, for example, type classes are not vectorized, and neither are lists. Furthermore, in a full implementation we will need support for partial vectorisation [16], among other things.

As a result, although all the examples in this paper should work when run sequentially, they may not all vectorise as written, even in GHC 6.10.

A good source of working code is in the Darcs repository http://darcs . haskell.org/packages/ndp, whose sub-directory examples/ contains many executable examples.

\subsection{Further reading}

Blelloch and Sabot originated the idea of compiling nested data parallelism into flat data parallelism [17], but an easier starting point is probably Blelloch subsequence CACM paper "Programming parallel algorithms" [9], and the NESL language manual [18]. 
Keller's thesis [15] formalized an intermediate language that models the central aspects of data parallelism, and formalized the key vectorisation transformation. She also studied array fusion, to eliminate unnecessary intermediate arrays. Leshchinskiy's thesis [8] extended this work to cover higher order languages ([19] gives a paper-sized summary), while Chakravarty and Keller explain a further generalization to handle user-defined algebraic data types [14].

Data Parallel Haskell is an ongoing research project [11]. The Manticore project at Chicago shares similar goals [20].

\section{References}

1. Mohr, E., Kranz, D.A., Halstead, R.H.: Lazy task creation - a technique for increasing the granularity of parallel programs. IEEE Transactions on Parallel and Distributed Systems 2(3) (July 1991)

2. Trinder, P., Loidl, H.W., Pointon, R.F.: Parallel and Distributed Haskells. Journal of Functional Programming 12(5) (July 2002) 469-510

3. Trinder, P., Loidl, H.W., Barry, E., Hammond, K., Klusik, U., Peyton Jones, S., Rebón Portillo, Á.J.: The Multi-Architecture Performance of the Parallel Functional Language GPH. In Bode, A., Ludwig, T., Wismüller, R., eds.: Euro-Par 2000 - Parallel Processing. Lecture Notes in Computer Science, Munich, Germany, 29.8.-1.9., Springer-Verlag (2000)

4. Prins, J., Chatterjee, S., Simons, M.: Irregular computations in fortran: Expression and implementation strategies. Scientific Programming 7 (1999) 313-326

5. O'Neill, M.: The genuine sieve of Eratosthenes. Submitted to JFP (2007)

6. Chakravarty, M., Keller, G., Lechtchinsky, R., Pfannenstiel, W.: Nepal - nested data-parallelism in haskell. In Sakellariou, Keane, Gurd, Freeman, eds.: Euro-Par 2001: Parallel Processing, 7th International Euro-Par Conference. Number 2150 in LNCS, Springer-Verlag (2001) 524-534

7. Barnes, J., Hut, P.: A hierarchical $O(n \log n)$ force calculation algorithm. Nature 324 (December 1986)

8. Leshchinskiy, R.: Higher-order nested data parallelism: semantics and implementation. PhD thesis, Technical University of Berlin (2006)

9. Blelloch, G.: Programming parallel algorithms. Communications of the ACM 39(3) (March 1996) 85-97

10. Leshchinskiy, R., Chakravarty, M., Keller, G.: Costing nested array codes. Parallel Processing Letters 12 (2002) 249-266

11. Chakravarty, M., Leshchinskiy, R., Jones, S.P., Keller, G.: Data Parallel Haskell: a status report. In: ACM Sigplan Workshop on Declarative Aspects of Multicore Programming, Nice (January 2007)

12. Schrijvers, T., Jones, S.P., Chakravarty, M., Sulzmann, M.: Type checking with open type functions. Submitted to ICFP'08 (2008)

13. Chakravarty, M., Keller, G., Peyton Jones, S.: Associated type synonyms. In: ACM SIGPLAN International Conference on Functional Programming (ICFP'05), Tallinn, Estonia (2005)

14. Chakravarty, M.M., Keller, G.: More types for nested data parallel programming. In: ACM SIGPLAN International Conference on Functional Programming (ICFP'00), Montreal, ACM Press (September 2000) 94-105 
15. Keller, G.: Transformation-based Implementation of Nested Data Parallelism for Distributed Memory Machines. PhD thesis, Technische Universite at Berlin, Fachbereich Informatik (1999)

16. Chakravarty, M.M., Leshchinskiy, R., Jones, S.P., Keller, G.: Partial vectorisation of Haskell programs. In: Proc ACM Workshop on Declarative Aspects of Multicore Programming, San Francisco, ACM Press (January 2008)

17. Blelloch, G., Sabot, G.: Compiling collection-oriented languages onto massively parallel computers. Journal of Parallel and Distributed Computing 8 (February 1990) $119-134$

18. Blelloch, G.: NESL: A nested data-parallel language (3.1). Technical Report CMUCS-95-170, Carnegie Mellon University (September 1995)

19. Leshchinskiy, R., Chakravarty, M.M., Keller, G.: Higher order flattening. In: Third International Workshop on Practical Aspects of High-level Parallel Programming (PAPP 2006). LNCS, Springer (2006)

20. Fluet, M., Rainey, M., Reppy, J., Shaw, A., Xiao, Y.: Manticore: A heterogeneous parallel language. In: ACM Sigplan Workshop on Declarative Aspects of Multicore Programming, Nice (January 2007) 